\title{
Research Article \\ Effects of Implementing Adaptable Channelization in Wi-Fi Networks
}

\author{
Abid Hussain ${ }^{1}$ and Nazar Abbas Saqib ${ }^{2}$ \\ ${ }^{1}$ School of Electrical Engineering and Computer Science, National University of Sciences and Technology (NUST), \\ Islamabad 44000, Pakistan \\ ${ }^{2}$ College of Electrical and Mechanical Engineering, National University of Sciences and Technology (NUST), \\ Islamabad 44000, Pakistan
}

Correspondence should be addressed to Abid Hussain; abid.hussain@seecs.edu.pk

Received 31 August 2015; Accepted 17 December 2015

Academic Editor: Pedro M. Ruiz

Copyright (C) 2016 A. Hussain and N. A. Saqib. This is an open access article distributed under the Creative Commons Attribution License, which permits unrestricted use, distribution, and reproduction in any medium, provided the original work is properly cited.

\begin{abstract}
The unprecedented increase of wireless devices is now facing a serious threat of spectrum scarcity. The situation becomes even worse due to inefficient frequency distribution protocols, deployed in trivial Wi-Fi networks. The primary source of this inefficiency is static channelization used in wireless networks. In this work, we investigate the use of dynamic and flexible channelization, for optimal spectrum utilization in Wi-Fi networks. We propose optimal spectrum sharing algorithm (OSSA) and analyze its effect on exhaustive list of essential network performance measuring parameters. The elementary concept of the proposed algorithm lies in the fact that frequency spectrum should be assigned to any access point (AP) based on its current requirement. The OSSA algorithm assigns channels with high granularity, thus maximizing spectrum utilization by more than $20 \%$ as compared to static width channel allocation. This optimum spectrum utilization, in turn, increases throughput by almost 30\% in many deployment scenarios. The achieved results depict considerable decrease in interference, while simultaneously increasing range. Similarly signal strength values at relatively longer distances improve significantly at narrower channel widths while simultaneously decreasing bit error rates. We found that almost $25 \%$ reduction in interference is possible in certain scenarios through proposed algorithm.
\end{abstract}

\section{Introduction}

The rapid development of wireless technologies has shifted the whole paradigm of networking. The majority of enterprises now prefer wireless networks over its wired counterpart, due to cost effectiveness, mobility feature, and minimal infrastructure requirements. Few years back, Wi-Fi networks were deployed for few users per AP scenarios. The advent of small hand held devices like smart phones, personal digital assistant (PDA) devices, and tablets has substantially increased the number and diversity of users connected simultaneously to the same AP. This large user base and assortment has exhaustively tested the efficiency of wireless networking protocols. The dense population of Wi-Fi devices has compelled network design engineers to optimize parameters like capacity, coverage, spectrum utilization and interference, and so forth which were more or less irrelevant some years ago.
In today's and future wireless networks, the most desired goal will probably be increased network capacity, minimal interference, and optimal spectrum utilization.

The scarcity of unlicensed wireless spectrum, used by Wi-Fi networks, and further sharing of this spectrum with other electronic appliances, like microwave ovens, cordless phones, children toy remotes, and so forth, make a strong case to effectively utilize the spectrum. However, static width channels [1] used for the communication of Wi-Fi networks led to the waste of this precious resource. The majority of today's wireless networking protocols fine-tune several communication parameters according to ambient conditions. However, quite surprisingly, Wi-Fi networks keep width of the transmission channel static, throughout the communication irrespective of environmental conditions. This static channelization causes underutilization of spectrum in several cases. To explain this claim, let us consider a network of 
three APs in close vicinity that are in interference range of each other. Different number of users is associated with these APs resulting in different throughput requirements of each AP. Let us consider that throughput requirements are $10 \mathrm{Mbps}$, $20 \mathrm{Mbps}$, and $30 \mathrm{Mbps}$ for AP 1, 2, and 3, respectively. According to normal static channel assignment scheme, all of these three APs communicate over same channel widths. It is a well-known fact that throughput is directly proportional to channel width [2]. In this case, AP 1 is underutilizing its spectrum share while AP 3 is starving. This phenomenon substantially decreases the efficiency of network. Therefore, considering the above explained facts, it is highly desired that physical layer protocols of spectrum utilization in Wi-Fi networks should be reevaluated and new protocols and standards must be implemented to ensure maximum utilization of available frequency resource.

This research work aims to evaluate the effect of flexible channelization on essential network performance measurement parameters. We propose a dynamic spectrum sharing algorithm that assigns channels of different widths based on environmental conditions and throughput requirements of an AP. Through series of experiments conducted on real testbed, the effect of OSSA on parameters like network capacity, throughput, spectrum utilization, interference management, bit error rate, delay spread, transmission range, and power consumption has been evaluated.

The public and enterprise networks became densely populated with the advent of bring your own device [3] concept. A large number of users at public places like shopping malls, airports, train stations, and stadiums use public hot-spots for their communications through hand held PDAs, smart phones, and tablets. Provision of sustained connectivity and quality of service (QoS) to this large and highly dense user base is a challenging task. The trivial design of conventional Wi-Fi networks like 820.11g [1] and its static channelization is not flexible enough to cope with the above described complex scenarios [2]. Therefore, to enhance the capacity of these networks, efficient spectrum utilization becomes critically important. Moreover, this high per unit area density of wireless clients requires a higher number of APs in close vicinity, to provide sustainable wireless connectivity. The benefit of deploying a higher number of APs per unit area is manifold, which includes a higher number of collision domains resulting in less average channel access time, higher signal strength values, higher network capacity, and better network coverage. However, increasing the number of APs in a small area causes interference. In this work, it has been observed that OSSA can solve both of these challenging tasks efficiently, by increasing network capacity by $30 \%$ and reducing interference by almost $25 \%$ in many cases.

As explained above, effective utilization of scarce frequency spectrum is highly desired matrix for performance enhancement of any wireless network. The static width channelization, employed in conventional Wi-Fi networks like $802.11 \mathrm{~b} / \mathrm{g}$ [1], does not help in accomplishment of this task. Some of current Wi-Fi standards like 802.11n and 802.11ac provide flexibility in channel width, through channel bonding [4]. However, channel bonding increases channel widths quite steeply and does not serve the purpose of effective spectrum utilization. The proposed algorithm adapts the channel width with high level of granularity, making it more efficient in achieving the goal of effective spectrum utilization. It has been observed that almost $20 \%$ increase in spectrum utilization is possible through OSSA.

In the majority of cases, the required throughput of smart devices like smart phones, PDAs, and tablets is substantially lower than the regular wireless devices like laptops and computer systems [5]. If required throughput is low, switching to a narrower channel is more beneficial, as narrower channels have high spectral efficiency, lower bit error rates, lower delay spread, and fading effects [6]. Narrower channels are also less susceptible to interference as compared to wider channels. Moreover, narrower channels have better SNR values at longer distances, as compared to wider width channels. In this paper, detailed analysis of effect of variable width channels on coverage of wireless networks is presented. It is observed, that a significant increase of almost 50\% in network coverage is achievable by using narrower width channels. Moreover, power consumption is a critically important factor in case of smart phones and PDAs. These devices have limited power supply, provided through a rechargeable battery. The width of transmission channel has a direct impact on power consumption. The cumulative energy consumption of $\mathrm{Wi}$ Fi usage increases as the width of transmission channel increases, resulting in rapid depletion of stored power. The situation becomes even worse, if required throughput is substantially low, which is true most of the times [7]. In this paper, we provide a detailed analysis of power consumption on different channel width.

The contribution of this research work can be outlined as follows:

(1) Development of a fine-grained, flexible channel assignment algorithm OSSA, for efficient spectrum utilization.

(2) Capacity, coverage, and throughput enhancement of Wi-Fi network through flexible channelization.

(3) Detailed analysis of impact of OSSA on power consumption.

(4) Interference, bit error rate, and delay spread analysis of OSSA.

The rest of this paper is organized as follows:

Section 2 explains flexible channelization in brief. Section 3 provides an overview of research reported in literature. Analytical modeling of interference and throughput of flexible width channelization are given in Section 4. We explain our proposed algorithm OSSA in Section 5 . Section 6 briefly describes the experimentation test-bed and methodology. A comprehensive discussion on achieved results is presented in Section 7. We have concluded our work in Section 8.

\section{Adaptable Channelization in Wi-Fi Networks}

Wi-Fi networks use two spectrum blocks for their communication. These blocks consist of $2.4 \mathrm{GHz}$ and $5 \mathrm{GHz}$ 
frequency ranges. In this work, we are emphasizing only $2.4 \mathrm{GHz}$ frequency spectrum used by $802.11 \mathrm{~b} / \mathrm{g} / \mathrm{n}$ networks for proof of concept purpose. However, the proposed flexible channel assignment algorithm is applicable to any spectrum block, with nominal changes in physical layer. The total available spectrum width in $802.11 \mathrm{~b} / \mathrm{g} / \mathrm{n}$ networks is divided into 14 channels of equal width of $22 \mathrm{MHz}$ each [1]. To minimize the effect of cochannels interference (CCI), a guard band of $5 \mathrm{MHz}$ is incorporated between any two consecutive channels. Despite guard band, the carrier frequencies of adjacent channels overlap with each other, which results in interference of signals on adjacent channels.

The frequency management organizations of different countries regulate the use of these channels in diverse manner, to minimize the effect of CCI. For example, the United States uses first 11 channels while Japan uses all 14 available channels for communication. In this study, we are discussing the case of 11 channels. Out of these 11 channels, channels 1,6 , and 11 are nonoverlapping and have minimum CCI. Each AP is configured on a different nonoverlapping channel than other AP, located in interference range of each other. If more than $3 \mathrm{APs}$ are required in a network, these channels are being reused. This reuse may cause interference, if APs are in interference range of each other. The standard implementation of $802.11 \mathrm{~b} / \mathrm{g}$ physical layer uses channels of static width. If throughput requirement of a specific AP is significantly low and associated nodes are dispersed in large area, this static channelization may cause spectrum underutilization. For resolution of this problem, the idea of adaptable channelization was first proposed in [2]. Managing frequency spectrum through flexible channelization affects almost all physical layer parameters, including but not limited to throughput, range, power consumption, interference, and bit error rate. To the best of our knowledge, a thorough evaluation of all these parameters for flexible channelization has not been presented. In this work, we evaluate the effect of flexible channelization on these essential communication parameters, by constructing channels of optimal width dynamically with respect to ambient conditions.

\section{Related Work}

The inspiring and continued growth of wireless networks has stimulated researchers and industry to bring improvement in these communication networks. Several techniques have been proposed and presented in literature, for enhancement of overall efficiency of these networks. Today's wireless networks, adjust quite a few transmission parameters like data rate, modulation and central frequency, and so forth during their transmission. A thorough discussion on bit rate selection and adaptation in wireless networks is presented in [8]. In this research work, authors presented SampleRate bit rate adaptation algorithm and evaluated how different bit rate selection on various wireless links in a network affect the achieved throughput. The authors in [9] thoroughly discussed the effect of modulation adaptation in wireless networks, by using relay nodes. In this work, it has been concluded that a significant throughput enhancement as compared to decode and forward based mechanism is possible, by using relay nodes that have better signal-to-nose ratio (SNR).

On the fly channel width adaptation is a relatively newer area of research which was first proposed in 2008 by work done in [2]. In this research, authors proposed SampleWidth algorithm, which dynamically adjusts the channel width based on throughput requirements. This algorithm uses four channel widths of 5, 10, 20, and $40 \mathrm{MHz}$. The authors used commodity WLAN network interface cards (NIC) and adapted the channel width by adjusting clock frequency of these NICs. They concluded that a substantial improvement in overall network throughput is achievable, by implementing SampleWidth algorithm. However, the drawback of this approach is priori channel width adjustment before actual transmission. Moreover, discrete and steep steps increase/decrease of channel width and limit the maximum possible advantage of width adaptation. The authors in [11] proposed a Fine-Grained Spectrum Allocation (FSA) algorithm that adapts channel width on per frame basis. However, adapting channel width on frame basis introduces huge amount of complexity and incur large switching delays. We found that switching of narrower to wider channels and vice versa should be minimal to achieve maximum performance benefit. In [12], the authors proposed a finegrained channel access mechanism (FICA) algorithm. FICA divides a transmission channel into several subchannels of varying widths and a node selects a subchannel according to its bandwidth requirement. This algorithm ensures several simultaneous transmissions in parallel.

In wireless networks interference management is of crucial importance. Extensive amount of research work has been published for effective management of interference in wireless networks. Most of this work aims to minimize interference for fixed channel widths. A game theory based interference reduction mechanism is proposed in [13]. This method exploits transmission power and scheduling of communication to minimize interference. Authors in this work conclude that game theoretic technique substantially decreases complexity and signaling overhead in interference management. Similar approaches of interference minimization have been proposed in [14-16]. Since all these techniques of interference minimization are primarily developed for standard implementation of physical layer, therefore these methods are not effective in case of adaptable channel width scenarios. To our knowledge, research presented in [17] is the only work that addresses the problem of interference in adaptable channel width algorithms. In this work, authors implemented FLUID algorithm, which models conflicts among transmitter/reciever pairs based on their SNR values. These conflict graphs are then used for transmission scheduling to minimize interference in adaptable channelization systems.

The number and density of Wi-Fi enabled devices have been greatly increased in the last decade due to considerable advancements in consumer electronics. Sustainable existence of this increasing density of wireless devices requires considerable amplification of network capacity. Numerous methodologies of capacity enhancements have been proposed in 
literature, among which [18-20] are of notable importance for this research work. In [18], authors evaluated network capacity with respect to node density. This work concludes that small number of user associated with APs having high SNR values substantially increases network capacity. Work presented in [19] explains how corelating locations of nodes affect network capacity. Although the majority of the networks follow poisson point distribution, in which each node's location is independent of other nodes, authors in this work modeled a special case, in which nodes are distributed in corelation to other nodes. In this work, the authors characterized the transmission capacity networks based on maximum spatial density of active links, given an outage constraint. The work presented in [20] explains the benefits of deploying multiple antennas on APs for capacity enhancements for an $N$ arbitrary nodes network with random node density. To our knowledge, no capacity enhancement technique through adaptable channelization has been discussed in literature.

A thorough survey of power consumption and range calculations, for both fixed and flexible channel width networks, is presented in $[2,10,21]$. Authors in [10] presented power consumption analysis of uploading and downloading of data in smart phones through Wi-Fi connection. It has been concluded that stored power of smart phones was depleted more readily if uploading and downloading data rate is high as compared to the power depletion at lower data rate. In [21], analysis of indoor range with varying channel separation is presented. Power consumption analysis for flexible channel widths is presented in [2]. Authors connected a resistor in series with the NIC power supply and measured the current passed through resistor. The power consumption analysis was made for 5, 10, 20, and $40 \mathrm{MHz}$ channel widths. The proposed work is different from already presented work in following ways:

(1) Implementing high level of granularity in adaptable channelization.

(2) Measurement and analysis of several unexplored performance parameters.

(3) Measurement of spectrum utilization.

(4) Implementation of adaptable channelization on more flexible USRP devices.

\section{Modeling Interference and Network Capacity}

In this section, we have extended the fixed widths models of network capacity and interference presented in literature to make them suitable for flexible channel widths.

4.1. Interference Modeling. Implementation of flexible channelization requires several communication factors to be addressed. One prominent factor among these factors is Cochannel interference (CCI). Since, by using channels of flexible widths, the component frequencies of channels change frequently, resulting in change of CCI. To address the issue of CCI in implementing OSSA, we analytically modeled it.

The standard implementation of Wi-Fi physical layer divides $22 \mathrm{Mhz}$ channel into 52 subcarriers. Four out of these subcarriers are used for the transmission of control traffic, while rest of the 48 subcarriers are used for data symbols [1]. The spreading of data symbols on 48 subcarriers uses orthogonal frequency division multiplexing (OFDM) or direct sequence spread spectrum (DSSS), for 802.11g and $802.11 \mathrm{~b}$ networks, respectively. The packing of 52 subcarriers in a single channel results in their overlap, both in the same channel and in adjacent channels. This overlapping results in cochannel interference (CCI). Authors in [22] evaluated CCI for fixed width channels as given in

$$
W_{i j}= \begin{cases}\sum 1-\left|F_{i}-F_{j}\right| c & \\ 0 & \text { Otherwise. }\end{cases}
$$

To extend this model for flexible channel widths, let us define some other parameters as follows:

(i) The channel width assigned to any $\mathrm{AP}_{i}=W$.

(ii) The interference measure between two adjacent $\mathrm{AP}_{i}$ and $\mathrm{AP}_{j}=I_{i j}$.

(iii) Number of nonoverlapping subcarriers $=N$.

(iv) Number of overlapping subcarriers $=K$.

(v) Number of overlapping subcarriers between channel $i$ and channel $j=X_{i j}$.

We can say that

$$
\begin{aligned}
& I_{i j} \propto X_{i j}, \\
& I_{i j}=c X_{i j},
\end{aligned}
$$

where $c$ is defined as overlapping constant that can be calculated by the number of overlapping subcarriers.

The desired objective is to achieve maximum channel width with minimal CCI. The numerical form of this optimization is given as

$$
\lim _{X_{i j} \rightarrow \min } X_{i j} \lim _{W \rightarrow W_{\max }} \sum_{K=1}^{K} X_{i j} \sum_{N=1}^{N} W_{i} .
$$

If all physical layer parameters remain constant, the throughput of a channel $i$ configured to $\mathrm{AP}_{i}$ is directly proportional to channel width $W_{i}$ and inversely proportional to its CII:

$$
\begin{aligned}
& T_{i} \propto W_{i}, \\
& T_{i}=Y W_{i} .
\end{aligned}
$$

Similarly,

$$
\begin{aligned}
T_{i} & \propto \frac{1}{I_{i}}, \\
T_{i} & =\frac{c}{I_{i}} .
\end{aligned}
$$


Since $W_{i}$ is a sum of nonoverlapping subcarriers $N_{i}$ and overlapping subcarriers $K_{i}$,

$$
W_{i}=\sum_{N=1}^{N} X_{N}+\sum_{K=1}^{K} I_{K}
$$

By combining (4) and (5),

$$
T_{i}=\frac{c Y W_{i}}{I_{i}}
$$

Substituting values for $W_{i}$,

$$
T_{i}=\frac{c Y}{I_{i}}\left[\sum_{N=1}^{N} X_{N}+\sum_{K=1}^{K} I_{K}\right]
$$

4.2. Network Capacity Modeling. Let us assume that there are $N$ nodes and $K$ access points in a network. These $K$ APs construct $K$ identical circles, in which their signal strength is greater than or equal to a certain threshold. Each AP is configured to use flexible channelization algorithm described in Section 5. The $N$ nodes are distributed randomly across these $K$ circles. From [19], it is given that the probability of any node $N_{i}$ belonging to a circle $K_{i}$ follows Poisson random variable with a probability distribution function of $\left[\operatorname{Pr} N_{i} \epsilon\right.$ $\left.K_{i}\right]=$ poisson $\left(\lambda_{i}\right)$. The objective function is to maximize $\sum_{i=1}^{n} T_{i}$ subject to $\sum_{i=1}^{n} T_{i}<C$, where $T_{i} \geq 0,1 \leq i \leq n$, where $T_{i}$ is the achieved throughput of user $i, n$ is the number of users sharing the radio resource in $K_{i}$ circle and $C$ is the capacity.

If the offered load on any $\mathrm{AP}_{i}$ is $L_{i}=\sum_{n=1}^{N} \sum_{k=1}^{K} n_{i k} r_{i k}$, where $n_{i} k$ is the load of any node $n_{i}$ on AP of ring $k$ and $r_{i} k$ is the total load of $i$ nodes on AP of ring $k$, then the objective function can be obtained by maximizing the utilization of frequency resource and minimizing interference. If currently $W$ width channel is available to an AP $K_{i}$, then the capacity enhancement will have to consider different scenarios. These scenarios are discussed as follows.

4.2.1. Scenario 1. Let $N$ uniformly distributed nodes be ready to send data to an AP $K_{i}$. If we assume that these $N$ nodes share available frequency resource equally with maximum data rate $R$ and have packet drop ratio PDR which is caused by interference of APs $K_{i}+1 \cdots k$ of adjacent rings, $R_{i}$ is a rate achieved by spreading signal over $X$ number of subcarriers; that is,

$$
R_{i}=\sum_{x=12}^{X} X_{i} r
$$

where $r$ is the transmission rate at a subcarrier:

$$
C=\max _{\left(R_{1}, \ldots, R_{N}\right)} \sum_{i=0}^{N}\left(1-\mathrm{PDR}_{i}\right) R_{i} .
$$

4.2.2. Scenario 2. Let $N$ uniformly distributed nodes be ready to send data to an AP $K_{i}$. If we assume that these $N$ terminals are allowed to transmit uniform amount of data at rate $R$ with a target amount of interference and resulting PDR, sharing available frequency resource equally, the capacity of this specific ring is given as

$$
C=\sum_{i=0}^{N}\left(\frac{1-\mathrm{PDR}_{i}}{\max _{\left(R_{1}, \ldots, R_{N}\right)} \sum_{i=0}^{N}\left(1 / R_{i}\right)}\right) .
$$

4.2.3. Scenario 3. Let us consider that $N$ wireless nodes are distributed uniformly and are ready to send data to an AP $K_{i}$. If we assume that each of these $N$ nodes has variable load and any node $n_{i}$ has $x_{i}$ amount of traffic to send at a data rate $R_{i}$ with a packet drop ratio PDR, the capacity of this specific ring is given as

$$
C=\max _{\left(R_{1}, \ldots, R_{N}\right)} \sum_{i=0}^{N}\left(\frac{1}{N}+\frac{x_{i}}{\sum_{i=1}^{N} x_{i}}\right)\left(1-\mathrm{PDR}_{i}\right) R_{i} .
$$

\section{The Optimal Spectrum Sharing Algorithm (OSSA)}

We propose a new algorithm of flexible channelization aiming to increase capacity, throughput, and spectrum utilization with minimal interference. The spectrum assignment to an AP is based on analytical calculations made in Section 4.

The OSSA adds channel flexibility by using different number of subcarriers for variable channel widths. For instance, a narrower channel will be constituted of less number of subcarriers as compared to a wider channel. A detailed overview of number of subcarriers per specific width channels and other corresponding physical layer parameters is given in Table 1

OSSA divides total transmission area into $K$ identical circles and places APs at the center of each circle. All APs have omnidirectional antennas, which make a circular transmission and interference radius around each AP. At network initialization phase, the four deployed APs use standard nonoverlapping channels. All APs are connected to a spectrum allocation and management server through wired links. This server manages the spectrum resources, based on calculation made in Sections 4.1 and 4.2. Two packet windows are being maintained at each access point. Let us name these windows as receiver window (RW) and transmitting window (TW). Both of these windows refresh their status after each 60-second time interval to remain updated. TW and RW helps in calculating the required uploading and downloading throughput of each $\mathrm{AP}$ at any given time. At central server, OSSA takes interference, signal strength (SS), and required throughput as inputs and calculates optimal channel width and number of subcarriers as output. The SS values are calculated through channel reciprocity [23]. If throughput requirement is changed at any instance, AP communicates this change to management server. If bandwidth requirement of an AP increases or decreases, it demands or releases spectrum resource accordingly. In case of spectrum demand, central management server checks status of available subcarriers that are not assigned to any other AP. OSSA running on central management server measures the impact of increase in channel width in terms 
TABLE 1: Number of subcarriers on different channel widths and frame transmission parameters.

\begin{tabular}{|c|c|c|c|c|c|c|c|c|c|c|c|}
\hline Channel width $(\mathrm{MHz})$ & 5 & 7.5 & 10 & 12.5 & 15 & 17.5 & 20 & 22.5 & 25 & 27.5 & 30 \\
\hline Number of subcarriers & 12 & 18 & 24 & 30 & 36 & 42 & 48 & 54 & 60 & 66 & 72 \\
\hline Symbol duration $(\mu \mathrm{S})$ & 16 & 12 & 8 & 7 & 6 & 5 & 4 & 3.5 & 3 & 2.5 & 2 \\
\hline $\operatorname{SIFS}(\mu \mathrm{S})$ & 40 & 30 & 20 & 17 & 15 & 13 & 10 & 8 & 6 & 5 & 4 \\
\hline DIFS $(\mu S)$ & 50 & 40 & 30 & 20 & 17 & 15 & 13 & 10 & 8 & 6 & 3 \\
\hline Slot duration $(\mu \mathrm{S})$ & 20 & 20 & 20 & 20 & 20 & 20 & 20 & 20 & 20 & 20 & 20 \\
\hline Guard interval $(\mu \mathrm{S})$ & 3.2 & 2.4 & 1.6 & 1.2 & 1 & 0.8 & 0.7 & 0.6 & 0.5 & 0.4 & 0.3 \\
\hline
\end{tabular}

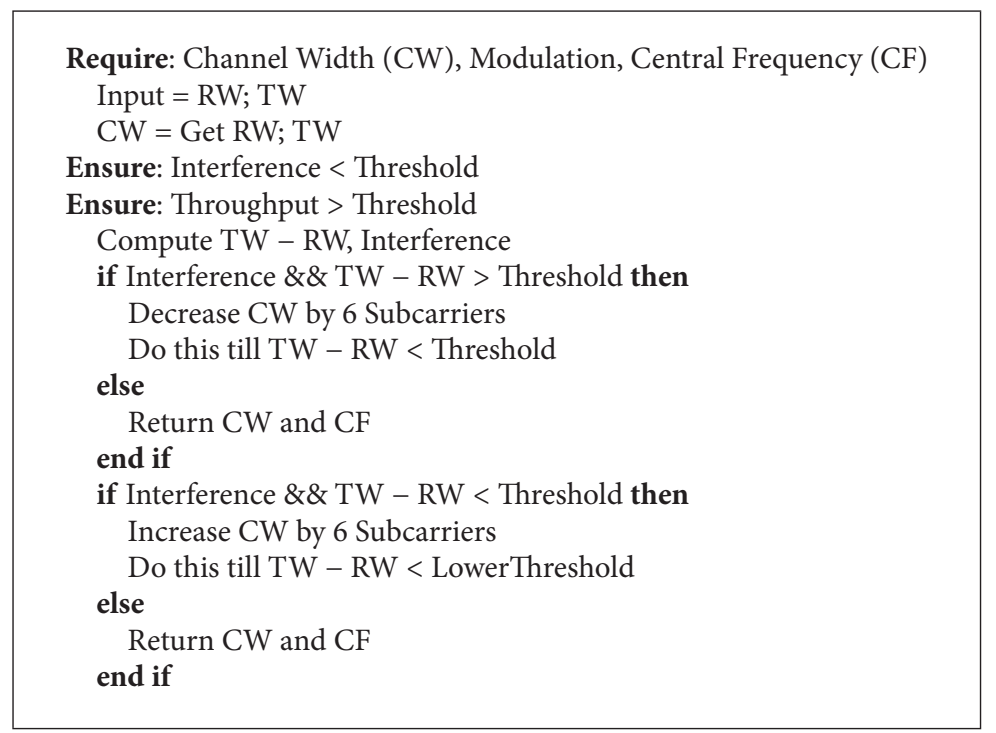

Algorithm 1: Channel width adaptation algorithm.

of throughput and interference. Based on threshold values of these measurements, management server decides about the increment of channel width. If subcarriers are available and subsequent change of increasing channel width keeps interference under a predefined threshold, then subcarrier frequencies are communicated to AP. After increase in channel width, AP starts spreading its signal to addition subcarrier frequencies in addition to already in-use subcarriers. If an AP releases spectrum resource, management server adds the subcarrier frequencies to available pool for on-demand dissemination to other APs. If throughput requirement of an AP decreases at any given time, it sends its new state of TW and RW to the management server. The management server in turn checks the prior in-use subcarriers and asks the AP to reduce its channel width by spreading its signal on lesser number of subcarrier frequencies.

In a case where cumulative spectrum demand exceeds the available, management server assigns channel width to achieve maximum network capacity with minimum interference. In this way, an AP having better SNR and lower interference index will use wider channel width and vice versa. Implementing flexible channelization required efficient width detection mechanisms at participating nodes. This detection mechanism involves the use of physical layer frame preamble. This technique is thoroughly discussed in [11].
Algorithm 1 presents our proposed algorithm based on best effort sharing of available frequency spectrum.

The use of flexible channelization in Wi-Fi networks affects several important physical layer parameters like central frequency, transmission range, achieved throughput, received signal strength values (RSS), delay spread and modulation schemes, and so forth. Since narrow channels have higher spectral efficiency and longer transmission range and are less susceptible to delay spread, the proposed algorithm recommends higher modulation schemes on these channels. In the majority of the cases this implementation increases the throughput to a great extent.

\section{Experimental Setup and Implementation}

For empirical evaluation of OSSA, we deployed an indoor network of three USRP kits connected to laptops running GNU radio software on Linux operating system (OS). A proof of concept implementation of OSSA for 802.11g wireless networks has been made by significantly modifying transceiver implementation provided at CGRAN (Comprehensive GNU Radio Archive Network) website [24] and better explained in [25]. This implementation is extendable to any 802.11 standard, by modifying its parameters at physical layer accordingly. A central management server constituting Dell 
TABLE 2: Confidence interval and statistical properties of throughput analysis.

\begin{tabular}{|c|c|c|c|c|c|c|c|c|c|c|c|c|}
\hline \multirow{3}{*}{$\begin{array}{l}\text { Measure } \\
\text { Algorithm } \\
\text { Channel wic }\end{array}$} & \multicolumn{12}{|c|}{ Throughput (Mbps) } \\
\hline & \multicolumn{6}{|c|}{ OSSA } & \multicolumn{6}{|c|}{ SampleWidth } \\
\hline & 5 & 10 & 15 & 20 & 25 & 30 & 5 & 10 & 15 & 20 & 25 & 30 \\
\hline Sample size & 8 & 8 & 8 & 8 & 8 & 8 & 8 & 8 & 8 & 8 & 8 & 8 \\
\hline Mean & 10.41 & 14.31 & 20.3 & 22 & 25.3 & 30 & 9.5 & 12.5 & 17.1 & 20.6 & 22.1 & 26.0 \\
\hline Standard deviation & 1.00 & 0.91 & 1.14 & 1.15 & 1.06 & 1.20 & 1.33 & 1.25 & 1.71 & 1.56 & 1.38 & 1.40 \\
\hline Sample variance & 2.06 & 0.84 & 1.3 & 1.33 & 1.14 & 1.44 & 1.78 & 1.56 & 2.93 & 2.43 & 1.91 & 1.97 \\
\hline Range & 3 & 2.4 & 3.2 & 3.4 & 2.8 & 3 & 3.3 & 3.4 & 4.3 & 4.1 & 3.7 & 4.1 \\
\hline Minimum & 8.7 & 13.1 & 18.5 & 20.3 & 23.7 & 28.5 & 7.9 & 10.9 & 15.1 & 18.4 & 20 & 23.7 \\
\hline Maximum & 11.7 & 15.5 & 21.7 & 23.7 & 26.5 & 31.5 & 11.2 & 14.3 & 19.4 & 22.5 & 23.7 & 27.8 \\
\hline Standard error & 0.35 & 0.32 & 0.40 & 0.40 & 0.37 & 0.42 & 0.47 & 0.44 & 0.60 & 0.55 & 0.48 & 0.49 \\
\hline Confidence level (95\%) & 0.83 & 0.76 & 0.95 & 0.96 & 0.89 & 1.0 & 1.11 & 1.0 & 1.43 & 1.30 & 1.15 & 1.17 \\
\hline
\end{tabular}

T-620 computer running OSSA on Linux OS has been placed for implementation of flexible channelization. Each USRP2 kit contained a $2400 \mathrm{RX} / \mathrm{TX}$ daughter card with omnidirectional antennas. The specifications of USRP kit and daughter cards are available at website [26]. Interference measurements have been made by implementing similar setup, constituting of four USRP2 kits acting as APs, attached to management server. There is a gigabit wired link between each AP and management server running OSSA on Linux OS. Two wireless enabled nodes are attached to each APs. The APs and the wireless nodes are located in an area of 200 $\times 200$ square feet. There is no interference of any other WiFi network operating in close vicinity. The physical layer of each AP is customized in such a way that an AP can switch to any of the narrower or wider channel widths at the end of current frame transmission. The wireless nodes detect the width of channels based on the preamble being transmitted by APs before the transmission of each frame. The deployed network diagram is given in Figure 1.

Detailed measurements of throughput enhancements, interference, bit error rates, power consumption, spectrum utilization, and transmission range have been made using deployed network. A thorough discussion on achieved results has been made in Section 7.

\section{Performance Results and Discussions}

A series of experiments have been performed to enumerate the affect of deploying OSSA on essential network performance parameters. The obtained results are averaged out by collecting traces of all APs for accurate efficiency measurement of OSSA. The confidence interval and allied statistical properties of achieved results of throughput, interference, and spectrum utilization are give in Tables 2, 3, and 4, respectively.

7.1. Spectrum Utilization. Increased spectrum utilization with minimized interference enhances network throughput and capacity. This calculation can be made by analyzing effect of spreading transmission signal over wide range of channel widths and constructing spectrogram of these channels.

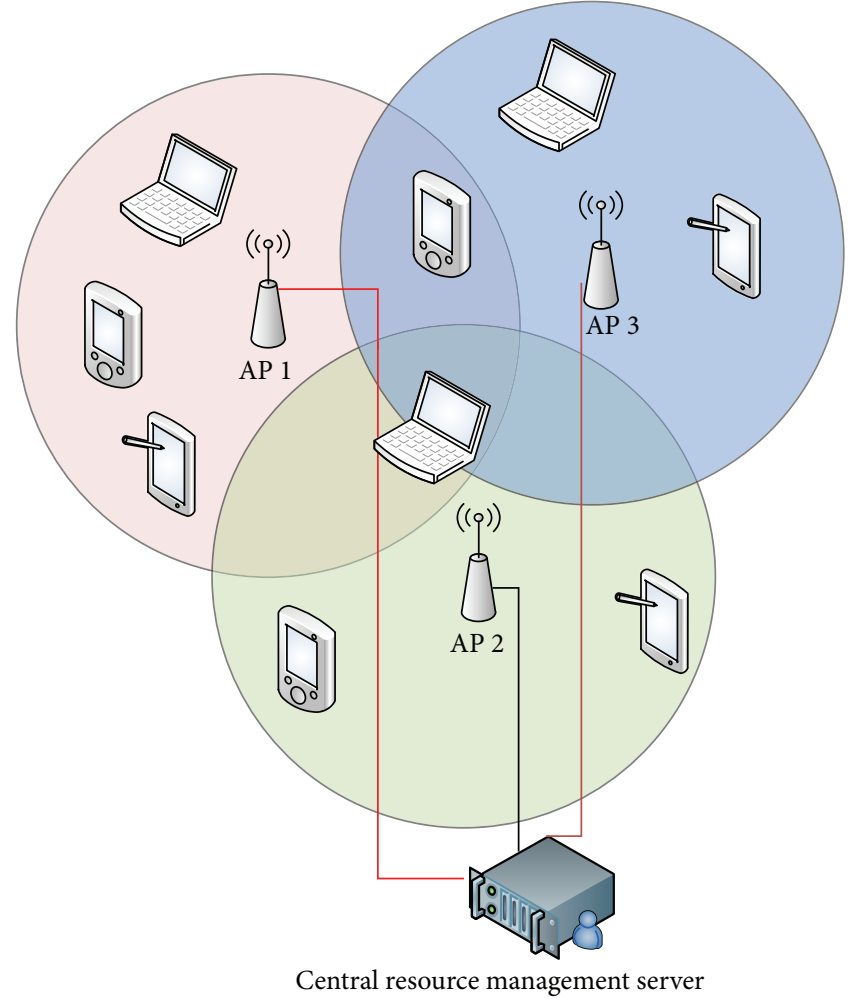

FIGURE 1: Architecture of deployed network.

Although this work does not focus on using standard fixed width channels, still spectrogram of standard channels can measure the efficiency of OSSA in spectrum utilization. Figure 2 depicts this spectrogram. The results presented in Figure 2 are gathered by using wifisurveyor Wi-Fi spectrum analyzer. These results show that spectrum utilization is significantly enhanced and nodes are spreading their signals even on overlapping channels. The use of overlapping channels, can be explained by the operational mechanism of OSSA. Since OSSA considers spectrum resource as combination of frequencies instead of discrete channels, these frequencies are assigned irrespective of channels. Therefore, 
TABLE 3: Confidence interval and statistical properties of interference analysis.

\begin{tabular}{lcccccccccccc}
\hline $\begin{array}{l}\text { Measure } \\
\text { Algorithm }\end{array}$ & \multicolumn{1}{c}{ OSSA } & \multicolumn{4}{c}{ Interference $(\mathrm{dBm})$} \\
\hline Offered load (Mbps) & 5 & 8 & 11 & 14 & 17 & 20 & 5 & 8 & 11 & 14 & 17 & 20 \\
Sample size & 8 & 8 & 8 & 8 & 8 & 8 & 8 & 8 & 8 & 8 & 8 & 8 \\
Mean & -28.93 & -27.42 & -25.35 & -17.03 & -15.02 & -12.15 & -26.08 & -25.17 & -24.45 & -23.98 & -20.48 & -25.41 \\
Standard deviation & 1.43 & 1.53 & 1.27 & 1.16 & 1.56 & 1.19 & 1.42 & 1.73 & 1.71 & 1.43 & 0.79 & 1.61 \\
Sample variance & 2.04 & 2.34 & 1.62 & 1.36 & 2.46 & 1.42 & 2.03 & 2.99 & 2.95 & 2.04 & 0.62 & 2.60 \\
Range & 3.8 & 4 & 3.4 & 2.9 & 4 & 3.2 & 4 & 4.4 & 4.4 & 4.3 & 2.4 & 4.1 \\
Minimum & -31 & -29.4 & -27.3 & -18.7 & -17.1 & -13.9 & -28.1 & -27.3 & $-26-5$ & -26.1 & -22 & -27.5 \\
Maximum & -27.2 & -25.4 & -23.9 & -15.8 & -13.1 & -10.7 & -24.1 & -22.9 & -22.1 & -21.8 & -19.6 & -23.4 \\
Standard error & 0.50 & 0.54 & 0.45 & 0.41 & 0.55 & 0.42 & 0.50 & 0.61 & 0.60 & 0.50 & 0.28 & 0.57 \\
Confidence level (95\%) & 1.19 & 1.27 & 1.06 & 0.97 & 1.31 & 0.99 & 1.19 & 1.44 & 1.43 & 1.19 & 0.66 & 1.34 \\
\hline
\end{tabular}

TABLE 4: Confidence interval and statistical properties of spectrum utilization.

\begin{tabular}{|c|c|c|c|c|c|c|c|c|c|c|c|c|}
\hline \multirow{3}{*}{$\begin{array}{l}\text { Measure } \\
\text { Algorithm } \\
\text { Offered load }\end{array}$} & \multicolumn{12}{|c|}{ Spectrum utilization (\%) } \\
\hline & \multicolumn{6}{|c|}{ OSSA } & \multicolumn{6}{|c|}{ SampleWidth } \\
\hline & 12 & 18 & 24 & 36 & 48 & 54 & 12 & 18 & 24 & 36 & 48 & 54 \\
\hline Sample size & 8 & 8 & 8 & 8 & 8 & 8 & 8 & 8 & 8 & 8 & 8 & 8 \\
\hline Mean & 49 & 59 & 62.12 & 71.12 & 81 & 94 & 40.25 & 50.25 & 57.37 & 62.25 & 70.25 & 75.12 \\
\hline Standard deviation & 2.39 & 2.39 & 2.53 & 2.03 & 2.16 & 1.30 & 1.90 & 1.90 & 1.92 & 1.66 & 2.43 & 2.16 \\
\hline Sample variance & 5.71 & 5.71 & 6.41 & 4.12 & 5.14 & 1.71 & 3.64 & 3.64 & 3.69 & 2.78 & 5.92 & 4.69 \\
\hline Range & 6 & 6 & 6 & 6 & 6 & 4 & 5 & 6 & 5 & 5 & 6 & 6 \\
\hline Minimum & 46 & 56 & 59 & 68 & 78 & 92 & 38 & 47 & 55 & 60 & 67 & 72 \\
\hline Maximum & 52 & 62 & 65 & 74 & 84 & 96 & 43 & 53 & 60 & 65 & 73 & 78 \\
\hline Standard error & 0.84 & 0.84 & 0.89 & 0.71 & 0.80 & 0.46 & 0.67 & 0.67 & 0.67 & 0.59 & 0.86 & 0.76 \\
\hline Confidence level (95\%) & 1.99 & 1.99 & 2.11 & 1.69 & 1.89 & 1.09 & 1.59 & 1.59 & 1.60 & 1.39 & 2.03 & 1.81 \\
\hline
\end{tabular}

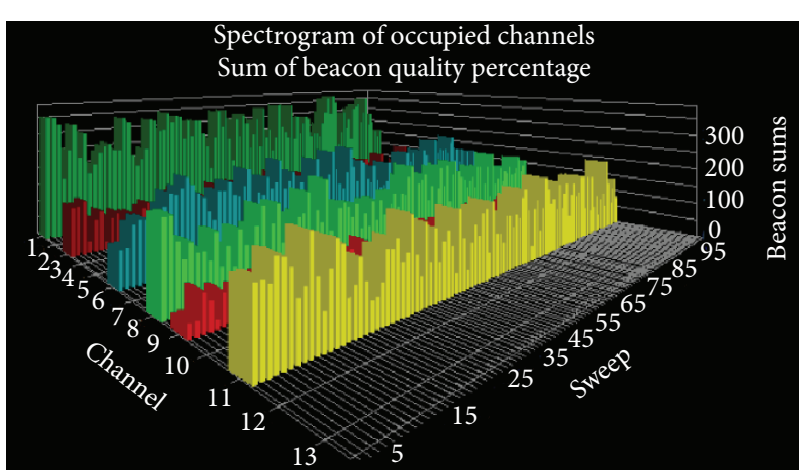

FIGURE 2: Channels spectrogram by deploying OSSA.

spectrogram shows use of overlapping channels in addition to standard nonoverlapping ones. Figure 3 enumerates the effect of implementing OSSA on $1,2,3$, and 4 APs in interference range of each other. It is appraised that if we use 4 APs, the spectrum utilization is more than $90 \%$. Since OSSA minimizes interference by implementing flexible channelization, it results in significantly increased spectrum utilization.

Figure 4 presents a comparison of spectrum use of OSSA with static and SampleWidth channel allocation schemes.

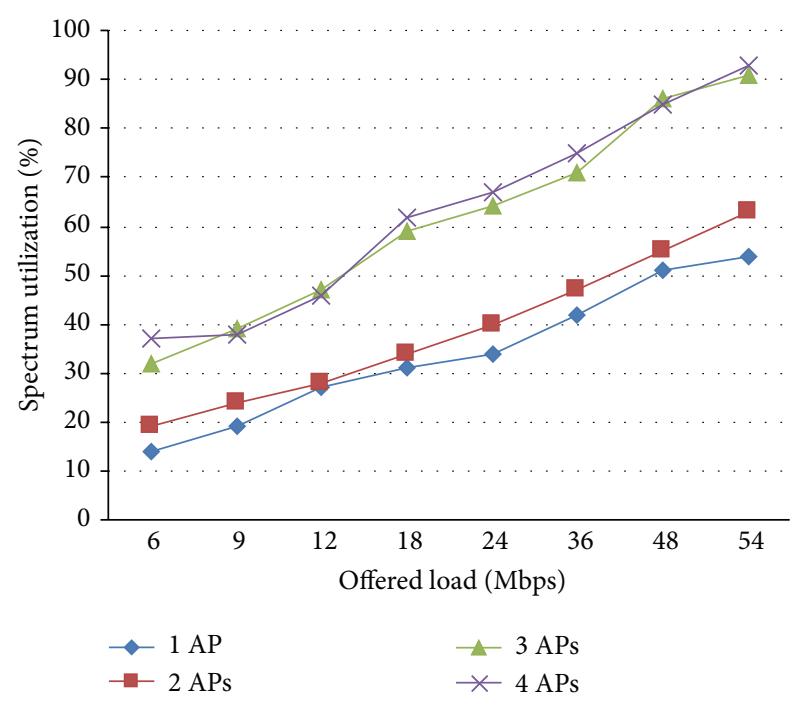

FIGURE 3: Spectrum usage by implementing OSSA on different number of APs.

It is noted that OSSA performs significantly better than its counterparts. This performance gain is due to the maximum utilization of subcarrier frequencies and high granularity in channel adaptation mechanism. 


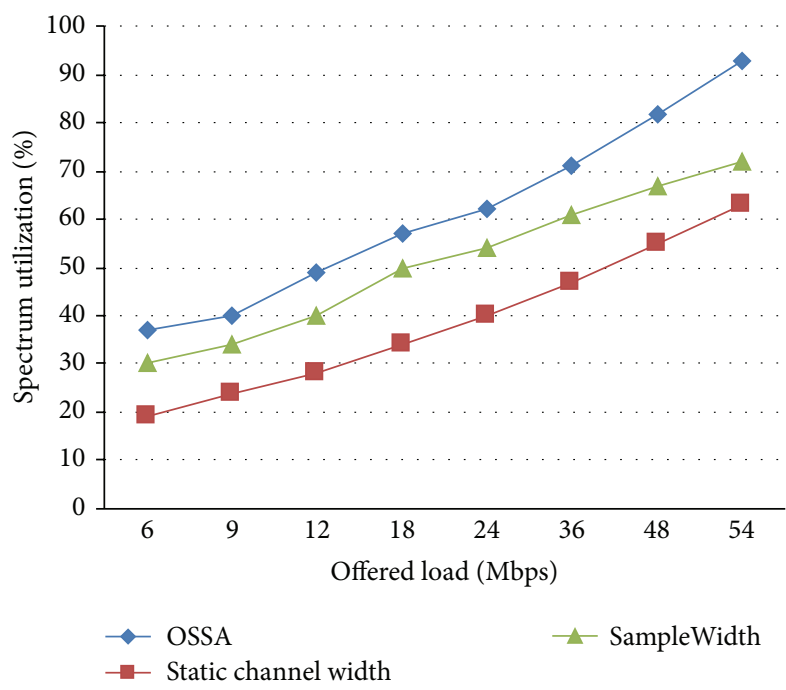

FIgURE 4: Comparison of spectrum usage.

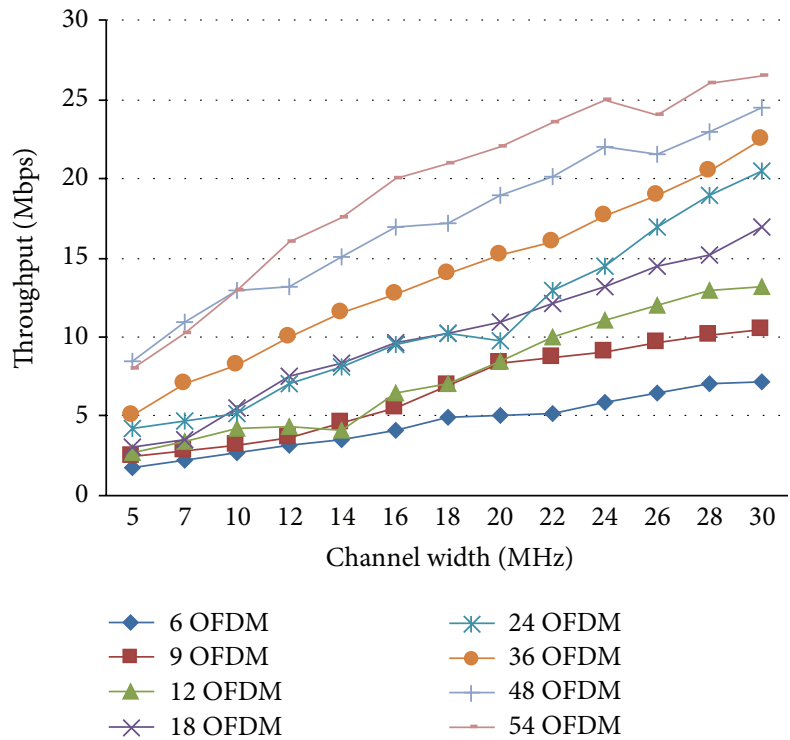

FIGURE 5: Effect of channel width with different modulations on TCP traffic.

7.2. Throughput Analysis. One of the most important factors in measuring network efficiency is achieved throughput. We have evaluated our proposed algorithm for throughput gains and compared the results with achieved throughput of standard implementation and with SampleWidth algorithm presented in [2]. The achieved throughput is averaged out on all APs for accurate calculation of network capacity enhancements.

Figures 5 and 6 present effects of channel width variations on TCP and UDP traffic by using different modulation schemes.

The results show that throughput does not have linear relationship with channel width. For example, in achieved results, throughput at channel width of $5 \mathrm{MHz}$ using 54 OFDM modulation is $8 \mathrm{Mbps}$, while width of $10 \mathrm{MHz}$ does

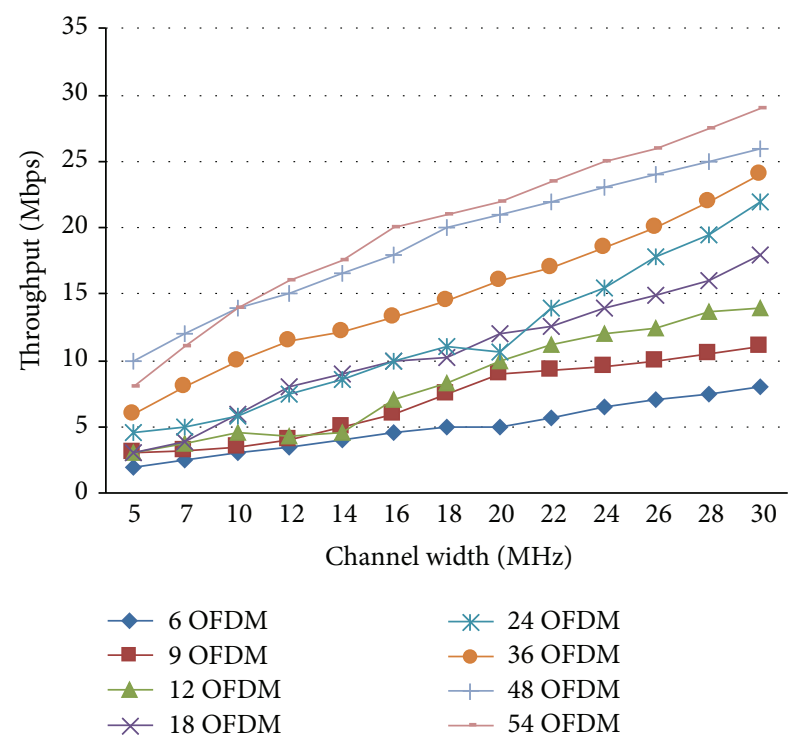

FIGURE 6: Effect of channel width with different modulations on UDP traffic.

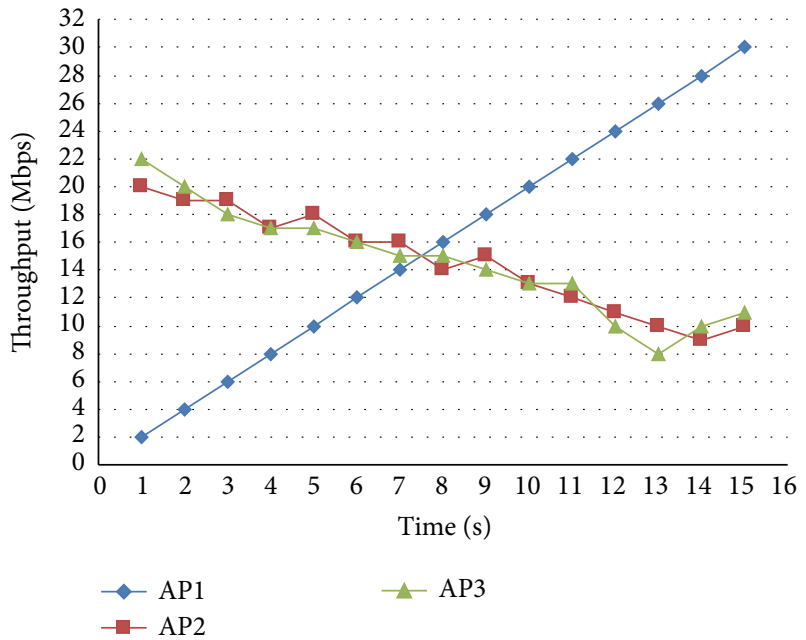

Figure 7: Throughput analysis when AP 1 is fed data rate while AP 2 and AP 3 are working in saturation mode.

not exactly double the throughput. We observed that wider channels are inherently more susceptible to interference, delay spread, and bit error rates resulting in decreased throughput. Another prominent factor involved in this behaviour is channel switching overhead. Moreover, preamble based detection mechanism is not very proficient in estimation of channel width which may result in frame losses due to inaccurate width detection.

Figures 7 and 8 show results of implementation of guaranteed data rate at different APs. We implemented OSSA by fixing data rate of one, two, and three participating APs in different experiments to evaluate its effect on other APs. These results can be used to evaluate the effect of OSSA in implementing quality of service classes. Figure 9 shows the results of OSSA when all participating APs are working in 


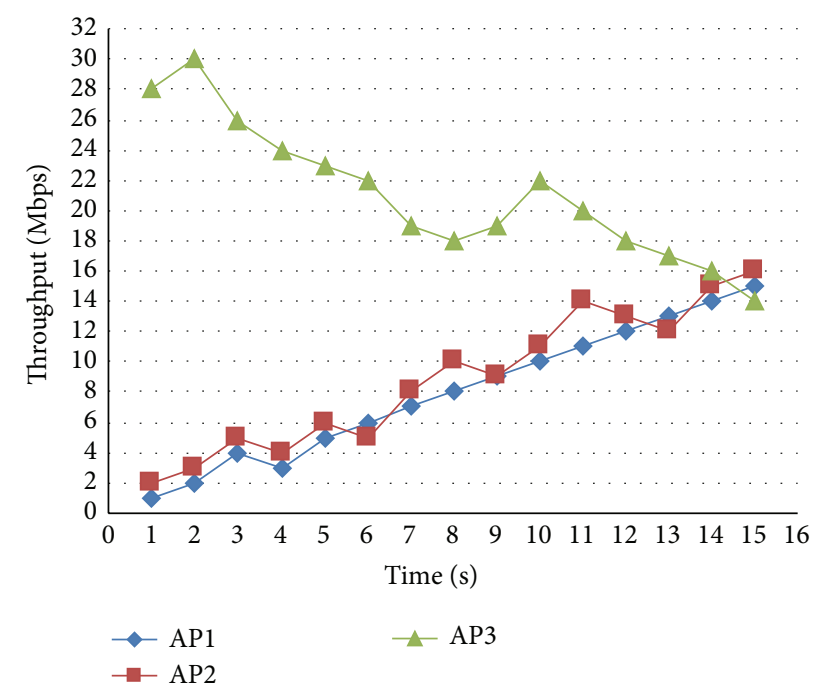

FIgURE 8: Throughput analysis when AP 1 and AP 2 are fed data rate and AP 3 is working in saturation mode.

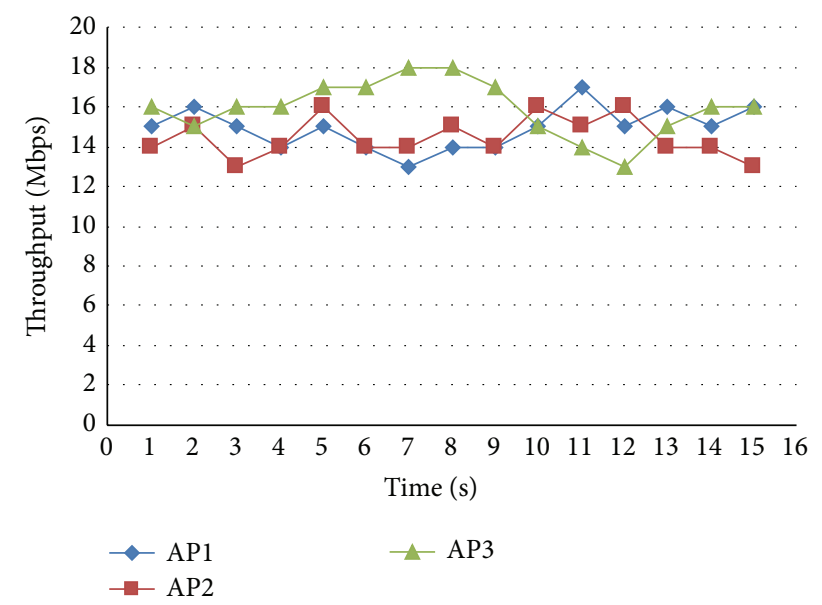

FIGURE 9: Throughput analysis when all APs are working in saturation mode.

saturated conditions. The achieved results show that available spectrum can be shared efficiently and fairly among APs by implementing OSSA. Figure 10 presents a comparison of OSSA with standard implementation and SampleWidth algorithms. From this comparison it is evident that OSSA outperforms the standard implementation and is also better than SampleWidth algorithm. This improvement in achieved throughput becomes almost equal to $30 \%$ at some points when compared with standard implementation. This high performance gain is a direct result of efficient and enhanced spectrum utilization which is a distinctive characteristic of OSSA over its contemporary techniques.

7.3. Analysis of Interference Management. Flexible channelization can play a vital role in interference management of wireless networks. Considering the importance of this aspect in performance of wireless networks, we carried out a detailed analysis of OSSA on interference management.

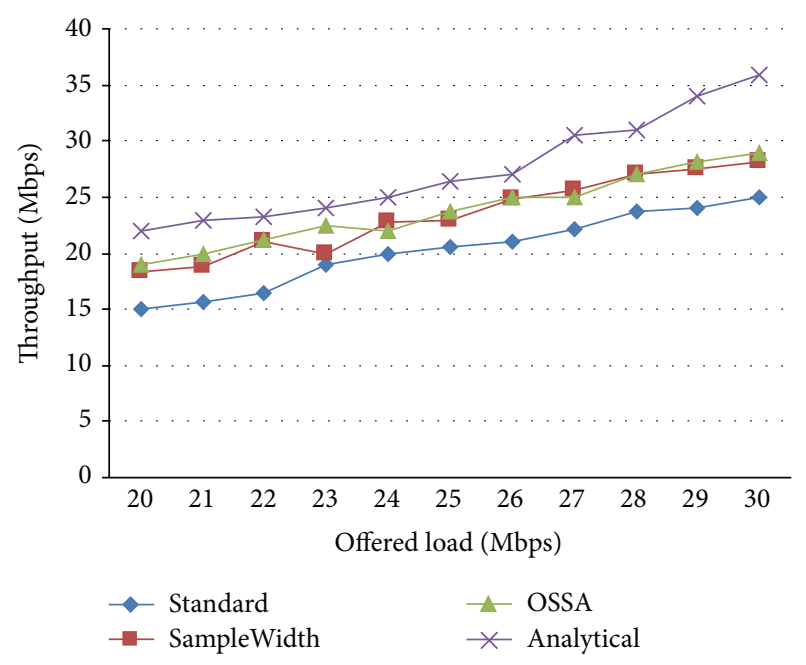

FIGURE 10: Throughput comparison with standard and SampleWidth algorithms [10].

Number of subcarriers

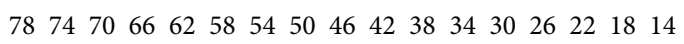

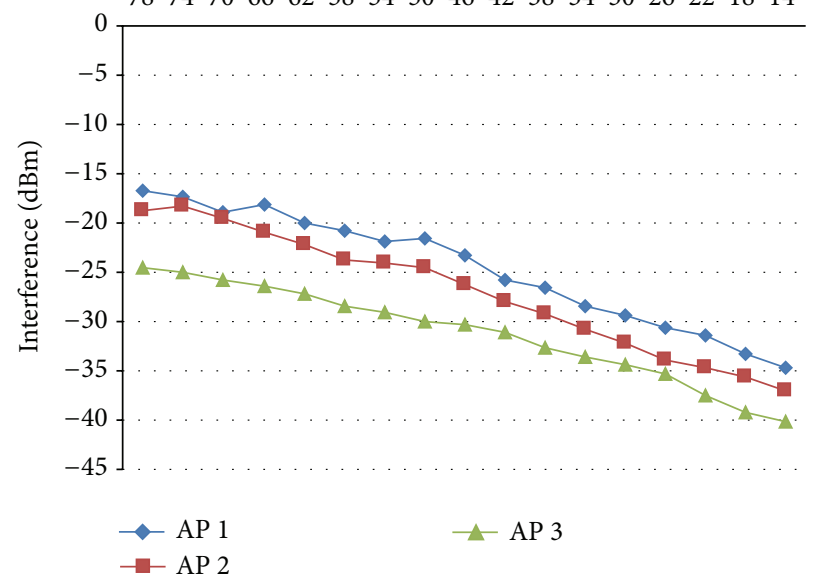

FIgURE 11: Channel width assigned to AP 1 is varied from $30 \mathrm{MHz}$ (78 subcarrier) to $5 \mathrm{MHz}$ (12 subcarriers) while channel widths of AP 2 and AP 3 are fixed at $20 \mathrm{MHz}$ (52 subcarriers).

This evaluation has been made by conducting series of experiments with different variations in number of APs, offered load, and required throughput.

Figures 11,12, and 13 demonstrate the interference calculations when flexible channelization is implemented in parallel to standard implementation while Figure 14 presents results when all APs are configured using flexible channelization.

The results presented in Figures 11 through 14 show that wider channel widths cause relatively high interference as compared to narrower widths. These results are quite obvious in a sense that a higher number of overlapping subcarriers increases interference. However, this effect can be minimized by employing flexible width channels to all APs across the network. The results of this setting are given in Figure 14. Implementation of OSSA on all APs in a network reduces interference by almost $25 \%$ as compared to static 


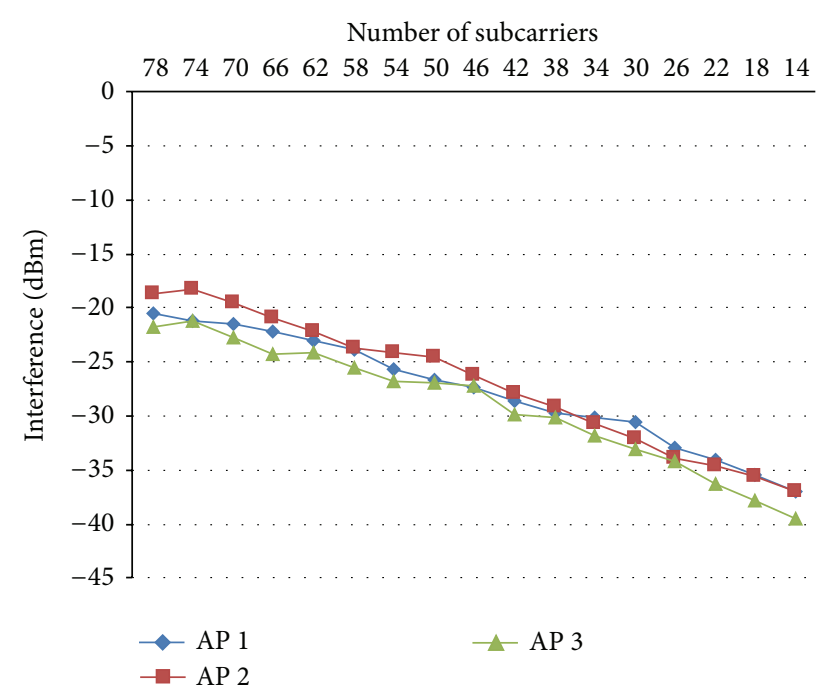

Figure 12: Channel width assigned to AP 2 is varied from $30 \mathrm{MHz}$ (78 subcarrier) to $5 \mathrm{MHz}$ (12 subcarriers) while channel widths of AP 1 and AP 3 are fixed at $20 \mathrm{MHz}$ (52 subcarriers).

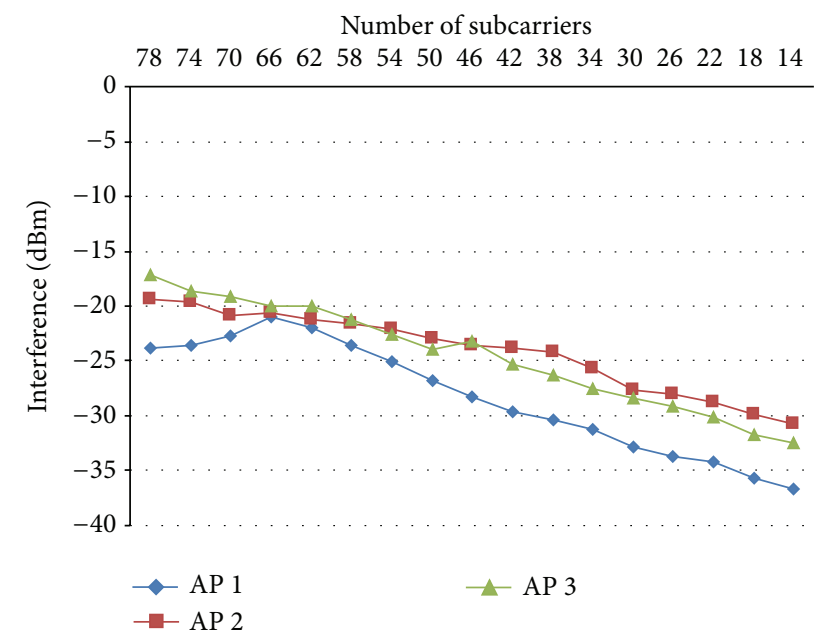

FIgURE 13: Channel width assigned to AP 3 is varied from $30 \mathrm{MHz}$ (78 subcarrier) to $5 \mathrm{MHz}$ (12 subcarriers) while channel widths of $\mathrm{AP} 1$ and $\mathrm{AP} 2$ are fixed at $20 \mathrm{MHz}$ (52 subcarriers).

width channel assignment. The comparison has been given in Figures 15 and 16. This decrease of interference is a result of adaptable subcarrier assignment to APs. When channel width of one AP increases, it triggers a subsequent decrease in channel width of adjacent $\mathrm{AP}(\mathrm{s})$. In this way, the spectrum resource is shared optimally with an added advantage of reduced interference.

Packing of flexible width channels is based on maximum separation of subcarrier frequencies. This maximum separation ensures that transmission channels are as far apart as possible. The maximum separation further reduces the interference. Figure 17 demonstrates the interference for OSSA implementation with maximum separation of frequencies.

As explained in Section 1, the density of wireless nodes is very high in public networks. This large user base can

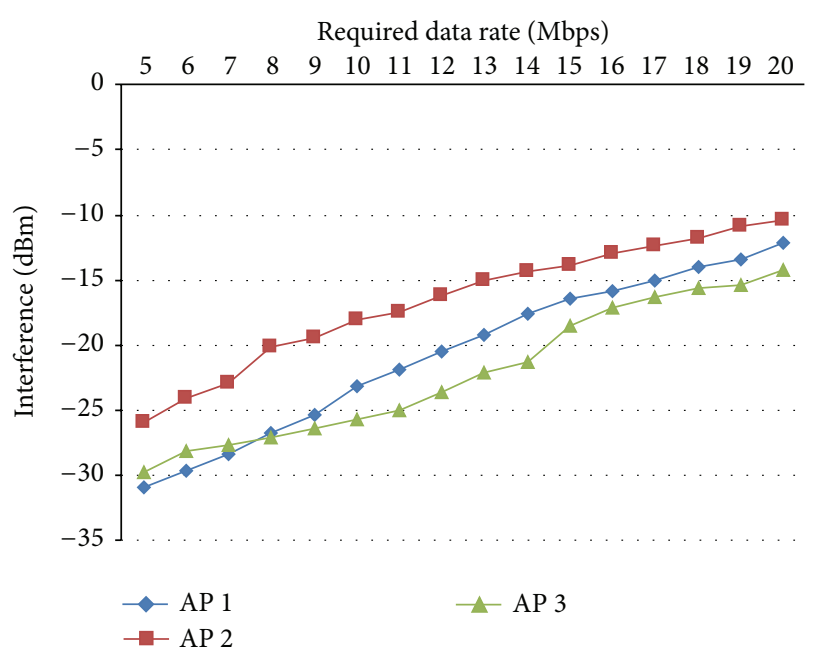

FIGURE 14: Interference calculations with employing varying number of subcarriers at different APs.

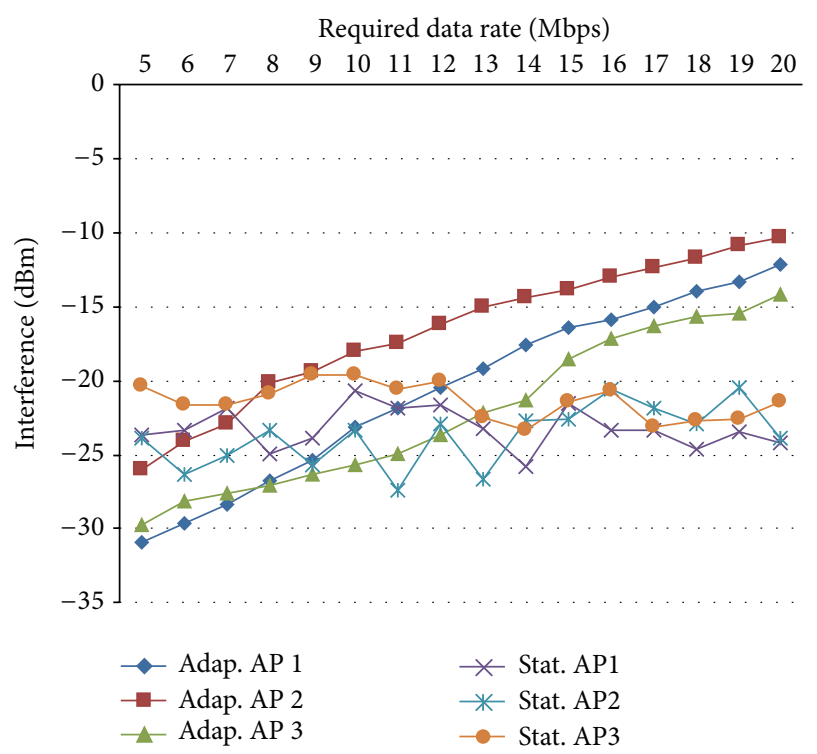

FIGURE 15: Interference comparison of OSSA with static channel assignment.

only be provided connectivity by deploying large number of APs. To assess the performance of OSSA implementation in such scenarios, we deployed four APs in interference range of each other. The results gathered are presented in Figure 18. For comparison purpose, we have also implemented standard channels to these APs in a different experiment. The results for standard implementation are given in Figure 19. The implementation of OSSA on these 4 APs can manage quite efficiently as compared to standard channel assignment. It is marked that OSSA optimally decreases the channel width of all APs in case of increase in interference over a threshold. Through this experiment, it is also evident that OSSA is scalable and efficient for networks with high AP density in a relatively small area. The insignificance in increase of number of APs is a result of automatic adjustment of channel 


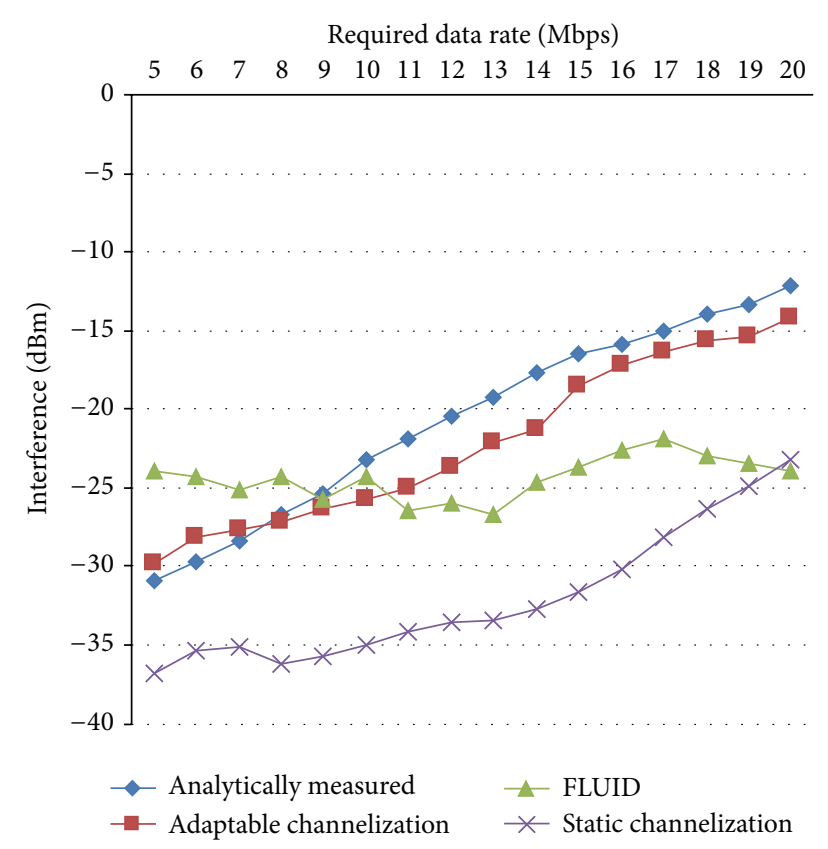

FIGURE 16: Interference comparison of measured, actual, and FLUID algorithm.

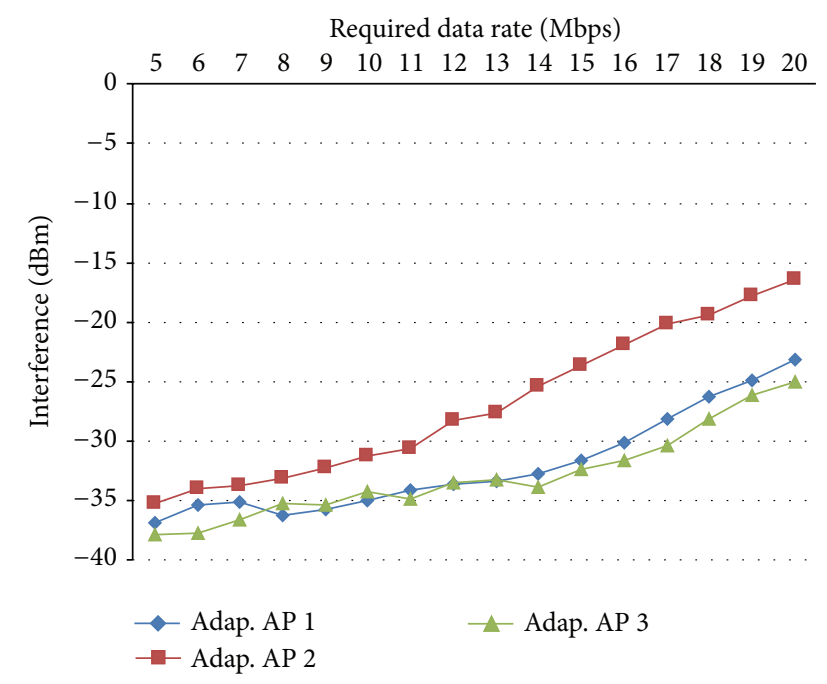

FIGURE 17: Interference for adaptable channel assignment having maximum separation of frequencies.

widths which reduces channels overlapping and consequent interference reduction.

7.4. Analysis of Bit Error Rate and Delay Spread Effect. The width of transmission channel has a significant impact on bit error rates. Wider channels employing higher order modulations incur high bit error rates compared to narrower channels with lower order modulations. The bit error calculations for different channel width employing varied modulation orders are given in Figure 20. No error correction or detection mechanisms are deployed and a single bit error in header, payload, or checksum can cause the frame to

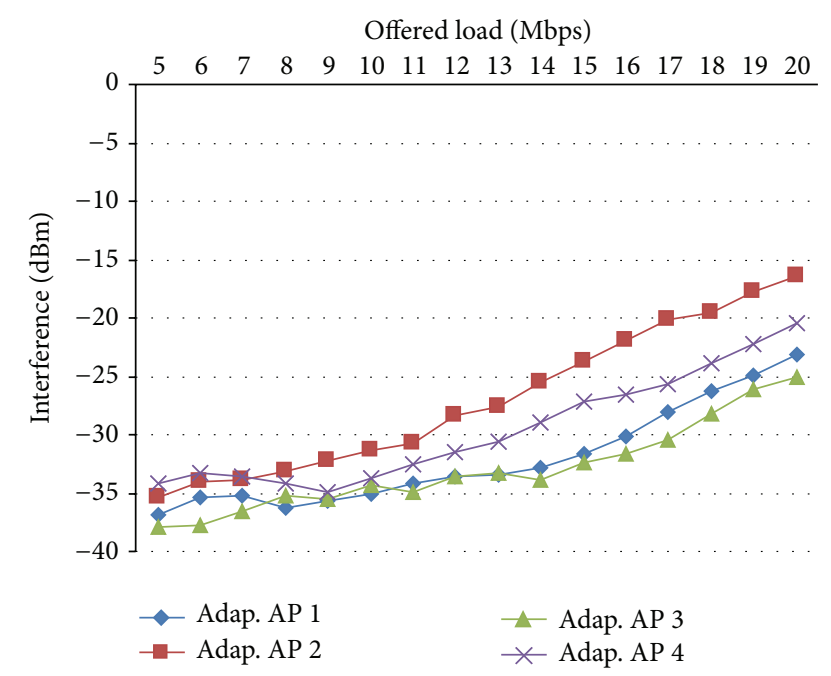

FIGURE 18: Interference when fourth AP is introduced with OSSA implementation.

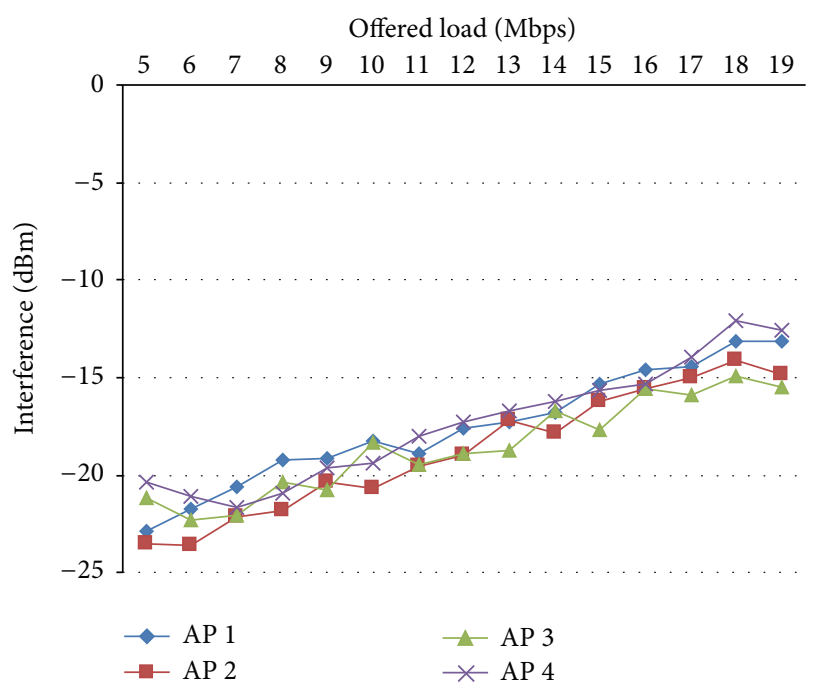

FIGURE 19: Interference when fourth AP is introduced in static channelization mode.

drop. The total sent and received frames are collected at all the nodes by using Wireshark packet sniffer. An exclusiveOR operation is performed for the dropped and originally transmitted frames to calculate the bit error. As shown in Figure 20, the wider channel widths and higher modulation schemes are more prone to errors. The reason behind the high BER is CCI and frame preamble based frequency detection as explained earlier.

For calculations of signal delay spread we have implemented a nanosecond counter on one AP. Since all the APs are placed in close proximity within the same room, therefore, it is assumed that delay spread is the same for each deployed AP. The time difference between the first and last received signals is calculated by using the nanosecond counter. Since wider channels have a higher number of allied frequencies, they experience more delay spread as compared to narrower 


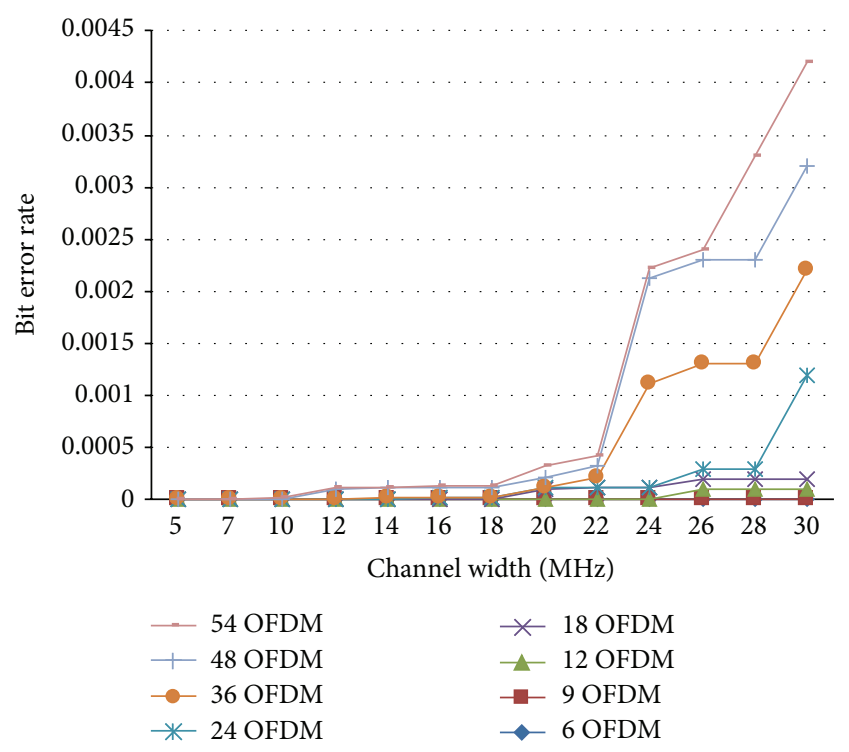

FIGURE 20: Bit error rate of channel widths using different modulation scheme.

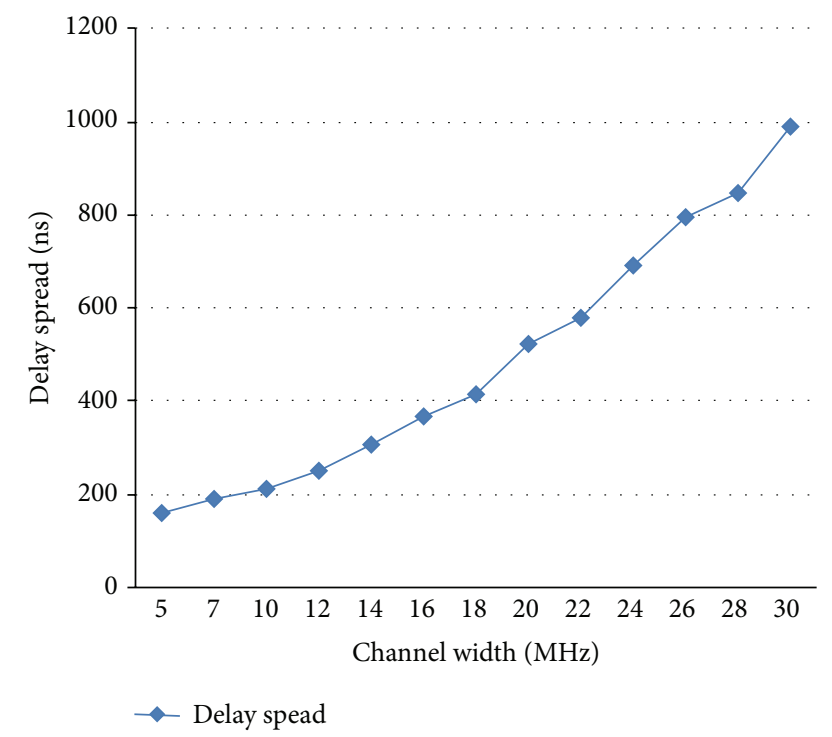

FIGURE 21: Delay spread on different channel widths.

channels. The effect of delay spread for different channel widths is given in Figure 21.

7.5. Analysis of Range and Power Consumption. Power consumption of Wi-Fi enabled devices is of critical importance especially in case of hand-held Wi-FI gadgets. For calculations of power consumption we connected the transceiver USRP daughter cards with digital power supply. The power consumption of standard WLAN adapter in transmitting mode ranges from $1400 \mathrm{~mW}$ for 3Com WLAN PCI Card with XJACK Antenna to $2750 \mathrm{~mW}$ for Cisco Aironet $350 \mathrm{WLAN}$ PCI adapter [27]. Effect of implementing OSSA on power consumption has been analyzed by varying power supply from $800 \mathrm{~mW}$ to $2800 \mathrm{~mW}$ for different channel widths and

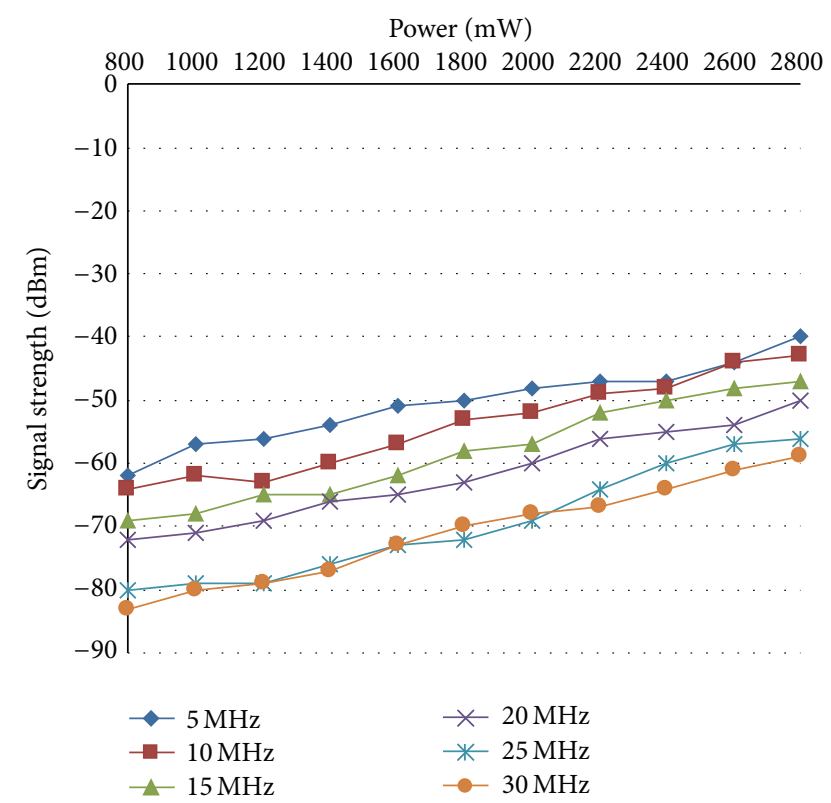

Figure 22: Power consumption and signal strength of different channel widths.

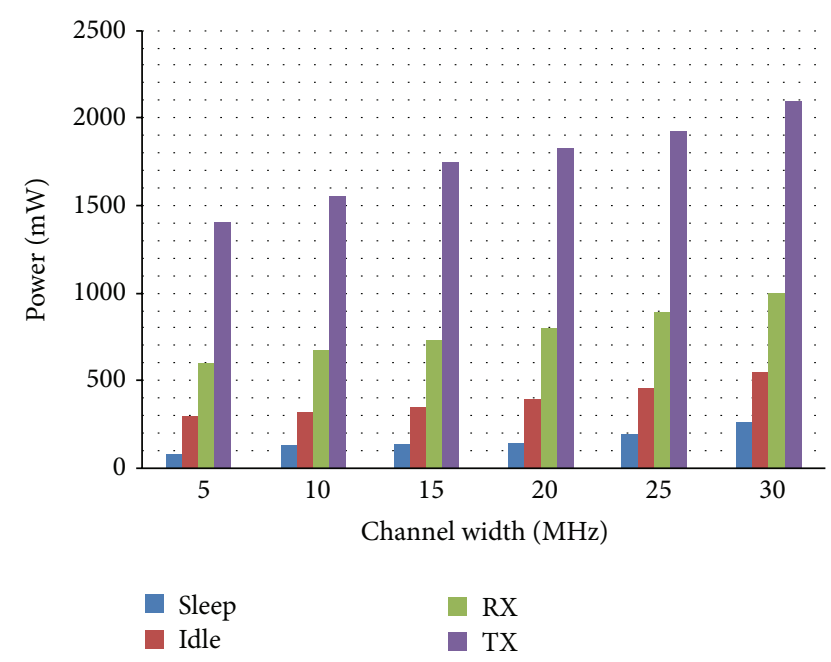

FIGURE 23: Power consumption in different operational modes.

SS has been measured at different transmission distances. The results obtained are given in Figures 22 and 23. The minimum power sensitivity of receiver antenna was $-96 \mathrm{dBm}$ (very low). From achieved results it is evident that we can save substantial amount of power by employing narrower channel widths without significantly decreasing SS. The reason behind this high power efficiency is high spectral efficiency of narrow bandwidth channels. Narrow channels require less amount of per $\mathrm{MHz}$ power and experience lower channel fading effects.

For calculations of transmission range of different channel widths, we performed experiments by placing connected nodes at different distances in both line of sight (LOS) and non-line of sight (NLOS) settlement. The SS of different channel widths has been measured to enumerate the effect of distance and obstacles in path of transmission signal. The 


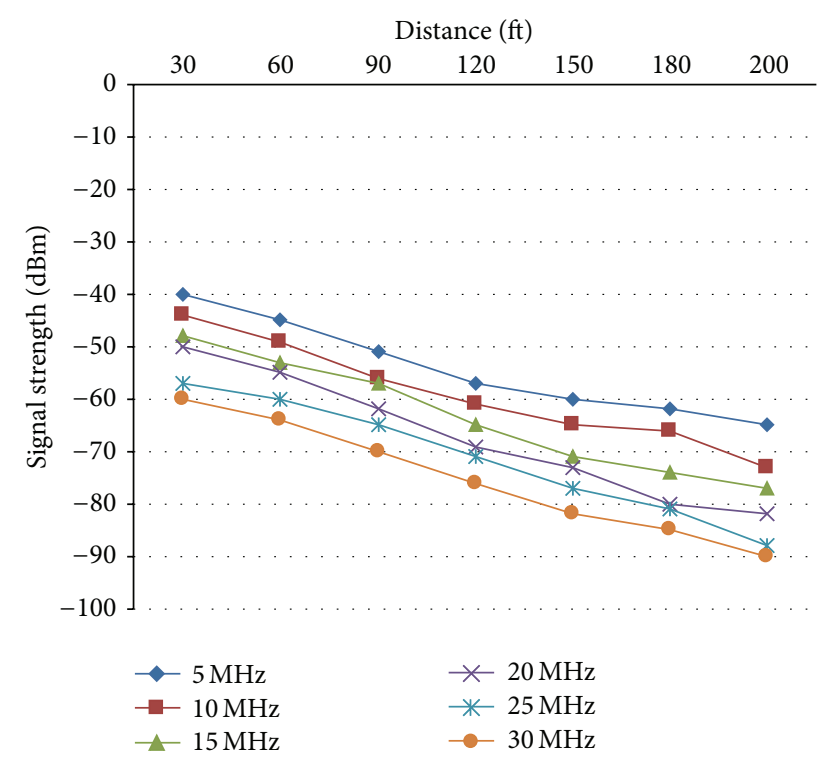

FIGURE 24: Signal strength at different distances for LOS communication.

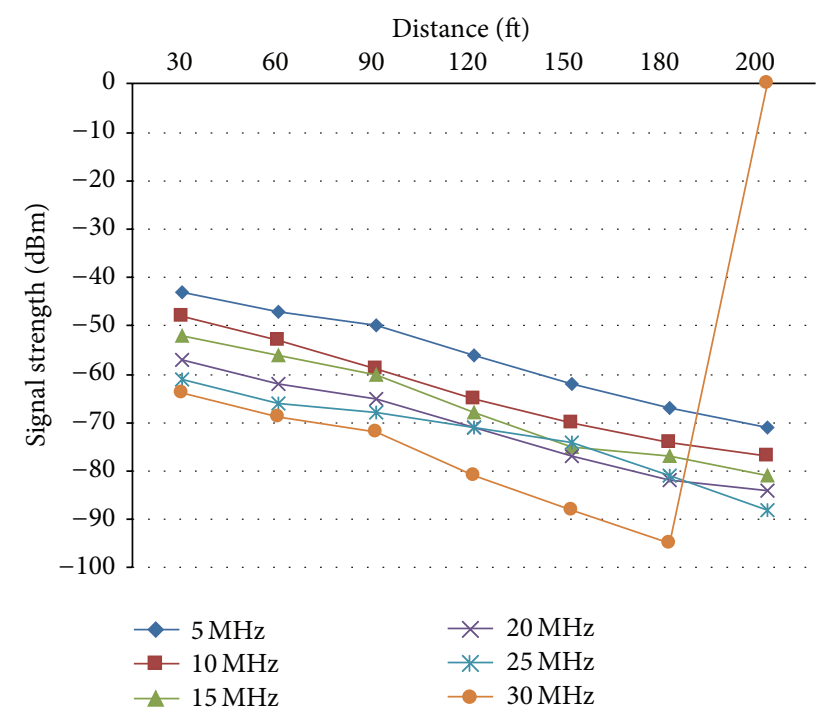

FIGURE 25: Signal strength at different distances for NLOS communication with one obstacle.

transmission power has been kept constant at $2000 \mathrm{~mW}$ for both LOS and NLOS settlements. The results for LOS communication are given in Figure 24. For calculation of NLOS communication for two scenarios (with one and two brick walls of 7-inche thickness each) between sender/receiver pair are given in Figures 25 and 26, respectively. The achieved results show that both transmission range and penetration capability of narrow band channels are significantly higher than wider width channels. This effect can be explained on the basis of per $\mathrm{MHz}$ available power and spectral characteristics of narrow and wide width channels. Since narrow width channels have more per $\mathrm{MHz}$ available power than wide width channels and have high spectral efficiency, therefore, better SS values are achieved. The vertical jumps of signal

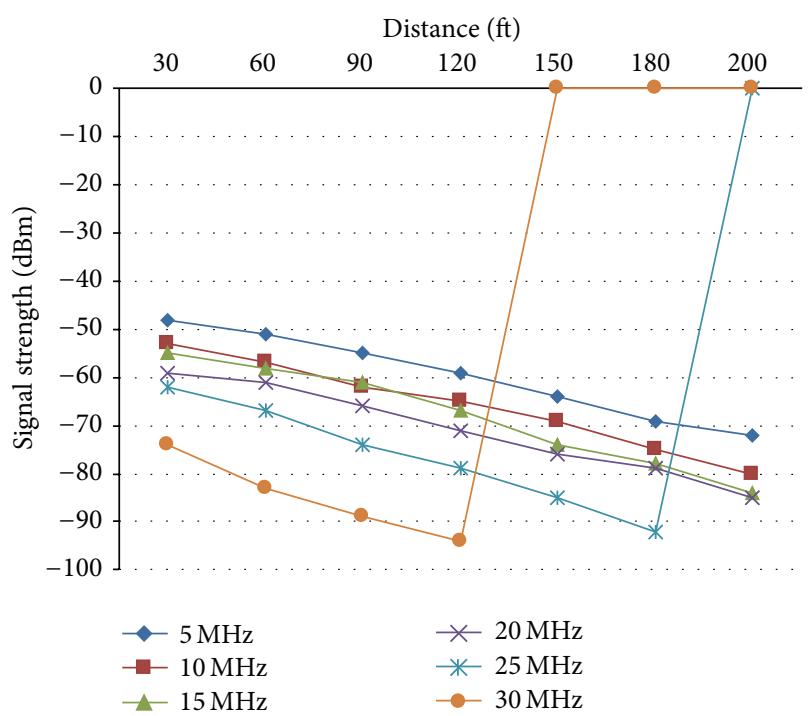

FIGURE 26: Signal strength at different distances for NLOS communication with two obstacle.

strength as given in Figures 25 and 26 are the points where no signal power is present.

\section{Conclusion}

In this work, we proposed an efficient flexible channel width adaptation algorithm, OSSA, that uses measures of interference, required throughput, and SS values to assign adaptable and variable width channels to APs. Thorough investigation through experimentation has been carried out to evaluate the affect of OSSA on essential physical layer parameters. These parameters include throughput, network capacity, interference, BER, spectrum utilization, power consumption, and transmission range. We reached a conclusion that OSSA uses available frequency spectrum optimally to achieve best possible network performance. It also adds benefit of channel width flexibility while simultaneously reducing interference. Achieved results show a significant improvement of almost $30 \%$ in achieved network capacity with different combinations of channel widths and interference measurements. It has also been observed that the proposed algorithm reduces interference by almost $25 \%$ when compared with interference of static channel assignment. It is concluded that the use of flexible width channels increase network capacity and reduces interference simultaneously, while both of these quantities are conflicting in standard implementation and a trade-off is required for optimal performance.

Future work includes the implementation of adaptable channel widths in MIMO based wireless networks like 802.11n and 802.11ac. Evaluation of effect of flexible channelization in $5 \mathrm{GHz}$ frequency spectrum is also required to be explored. In our opinion, implementation of flexible channelization has considerable potential in $5 \mathrm{GHz}$ range due to availability of higher number of transmission channels. 


\section{Conflict of Interests}

The authors declare that there is no conflict of interests regarding the publication of this paper.

\section{References}

[1] IEEE, "IEEE standard for information technology-specific requirements-part 11: wireless lan Medium Access Control (MAC) and Physical Layer (PHY) specifications," IEEE Std 802.11-2007 (Revision of IEEE Std 802.11-1999), 2007.

[2] R. Chandra, R. Mahajan, T. Moscibroda, R. Raghavendra, and P. Bahl, "A case for adapting channel width in wireless networks," in Proceedings of the ACM SIGCOMM Conference on Data Communication (SIGCOMM '08), pp. 135-146, ACM, Seattle, Wash, USA, August 2008.

[3] A. Armando, G. Costa, L. Verderame, and A. Merlo, "Bring your own device, securely," in Proceedings of the 28th Annual ACM Symposium on Applied Computing (SAC '13), pp. 18521858, Coimbra, Portugal, March 2013.

[4] W. Sun, O. Lee, Y. Shin et al., "Wi-Fi could be much more," IEEE Communications Magazine, vol. 52, no. 11, pp. 22-29, 2014.

[5] H. Falaki, R. Mahajan, S. Kandula, D. Lymberopoulos, R. Govindan, and D. Estrin, "Diversity in smartphone usage," in Proceedings of the 8th International Conference on Mobile Systems, Applications, and Services (MobiSys '10), pp. 179-194, ACM, San Francisco, Calif, USA, June 2010.

[6] K. Chintalapudi, B. Radunovic, V. Balan et al., "WiFi-NC: WiFi over narrow channels," in Proceedings of the 9th USENIX Conference on Networked Systems Design and Implementation (NSDI '12), USENIX Association, San Jose, Calif, USA, April 2012.

[7] N. Balasubramanian, A. Balasubramanian, and A. Venkataramani, "Energy consumption in mobile phones: a measurement study and implications for network applications," in Proceedings of the 9th ACM SIGCOMM Conference on Internet Measurement Conference (IMC '09), pp. 280-293, ACM, Chicago, Ill, USA, November 2009.

[8] J. C. Bicket, Bit-rate selection in wireless networks [Ph.D. thesis], Massachusetts Institute of Technology, 2005.

[9] Y. Zhang, Y. Ma, and R. Tafazolli, "Modulation-adaptive cooperation schemes for wireless networks," in Proceedings of the IEEE 67th Vehicular Technology Conference (VTC-Spring '08), pp. 1320-1324, Singapore, May 2008.

[10] A. Carroll and G. Heiser, "An analysis of power consumption in a smartphone," in Proceedings of the USENIX Conference on USENIX Annual Technical Conference (USENIXATC '10), vol. 14, p. 21, Boston, Mass, USA, June 2010.

[11] S. Yun, D. Kim, and L. Qiu, "Fine-grained spectrum adaptation in wifi networks," in Proceedings of the 19th Annual International Conference on Mobile Computing \& Networking (MobiCom '13), pp. 327-338, ACM, New York, NY, USA, October 2013.

[12] J. Fang, K. Tan, Y. Zhang et al., "Fine-grained channel access in wireless LAN," IEEE/ACM Transactions on Networking, vol. 21, no. 3, pp. 772-787, 2013.

[13] D. Gesbert, S. G. Kiani, A. Gjendemsj, and G. E. Ien, "Adaptation, coordination, and distributed resource allocation in interference-limited wireless networks," Proceedings of the IEEE, vol. 95, no. 12, pp. 2393-2409, 2007.

[14] D. López-Pérez, A. Valcarce, G. de La Roche, and J. Zhang, "OFDMA femtocells: a roadmap on interference avoidance," IEEE Communications Magazine, vol. 47, no. 9, pp. 41-48, 2009.
[15] J. Huang, R. A. Berry, and M. L. Honig, "Distributed interference compensation for wireless networks," IEEE Journal on Selected Areas in Communications, vol. 24, no. 5, pp. 1074-1084, 2006.

[16] U. Paul, A. Kashyap, R. Maheshwari, and S. R. Das, "Passive measurement of interference in wifi networks with application in misbehavior detection," IEEE Transactions on Mobile Computing, vol. 12, no. 3, pp. 434-446, 2013.

[17] S. Rayanchu, V. Shrivastava, S. Banerjee, and R. Chandra, "FLUID: improving throughputs in enterprise wireless LANs through flexible channelization," IEEE Transactions on Mobile Computing, vol. 11, no. 9, pp. 1455-1469, 2012.

[18] P. Gupta and P. R. Kumar, "The capacity of wireless networks," IEEE Transactions on Information Theory, vol. 46, no. 2, pp. 388404, 2000.

[19] R. K. Ganti, J. G. Andrews, and M. Haenggi, "High-SIR transmission capacity of wireless networks with general fading and node distribution," IEEE Transactions on Information Theory, vol. 57, no. 5, pp. 3100-3116, 2011.

[20] W.-Y. Shin, S.-W. Jeon, N. Devroye et al., "Improved capacity scaling in wireless networks with infrastructure," IEEE Transactions on Information Theory, vol. 57, no. 8, pp. 5088-5102, 2011.

[21] S. Sendra, M. García Pineda, C. Turró Ribalta, and J. Lloret, "Wlan ieee $802.11 \mathrm{a} / \mathrm{b} / \mathrm{g} / \mathrm{n}$ indoor coverage and interference performance study," International Journal on Advances in Networks and Services, vol. 4, no. 1, pp. 209-222, 2011.

[22] M. Zafer and E. Modiano, "Optimal adaptive data transmission over a fading channel with deadline and power constraints," in Proceedings of the 40th Annual Conference on Information Sciences and Systems (CISS '06), pp. 931-937, IEEE, Princeton, NJ, USA, March 2006.

[23] E. G. Larsson, O. Edfors, F. Tufvesson, and T. L. Marzetta, "Massive MIMO for next generation wireless systems," IEEE Communications Magazine, vol. 52, no. 2, pp. 186-195, 2014.

[24] The Comprehensive GNU Radio Archive Network (CGRAN), IEEE 802.11, OFDM, IEEE 802.11a/g/p ofdm transceiver.

[25] A. Costantini, Implementation of an IEEE 802.11p transmitter in open-source Software Defined Radio [M.S. thesis], Università del Salento, Lecce, Italy, 2008-2009.

[26] Ettus Research, Universal Software Radio Peripheral and Daughter Boards, http://www.ettus.com/product/details/UN210KIT.

[27] S. Chiaravalloti, F. Idzikowski, and L. Budzisz, "Power consumption of wlan network elements," Tech. Rep. TKN-11-002, Technical University of Berlin, Berlin, Germany, 2011. 

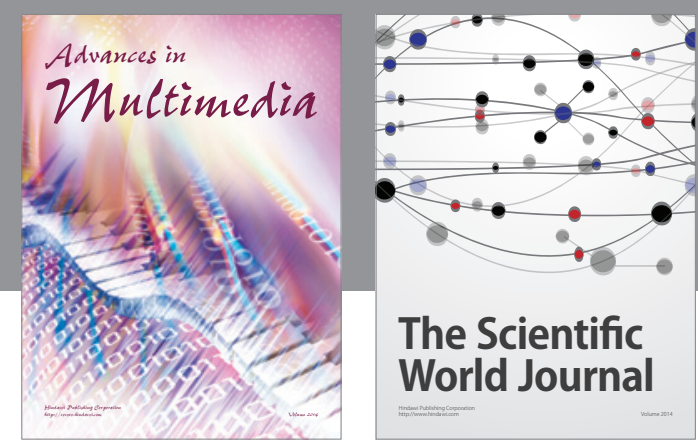

The Scientific World Journal
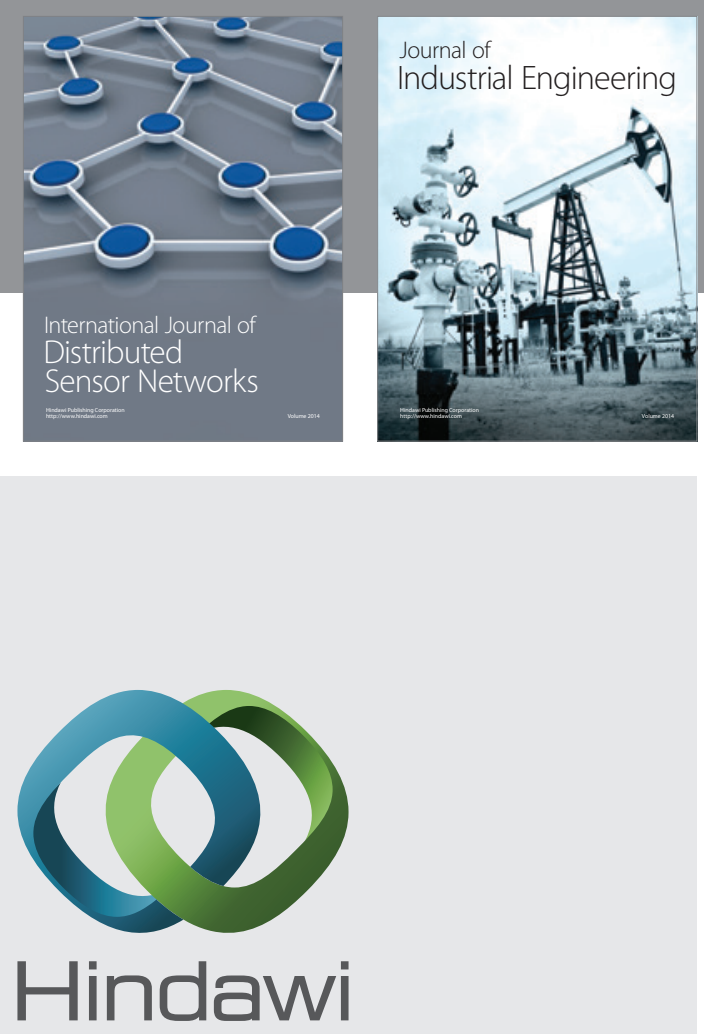

Submit your manuscripts at

http://www.hindawi.com

\section{Computer Networks} and Communications
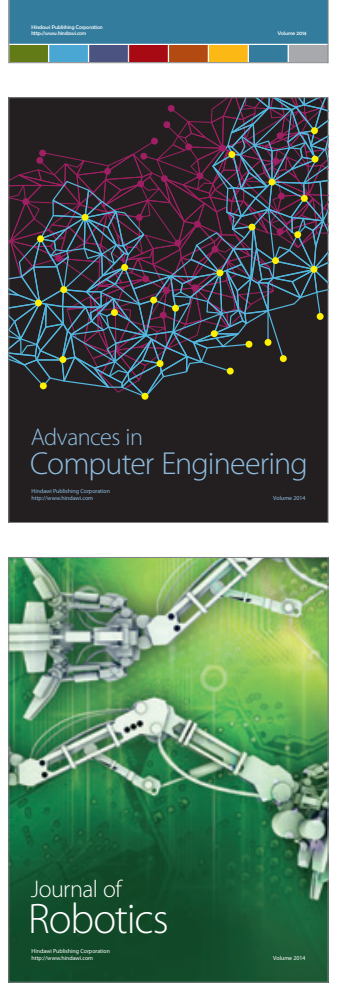
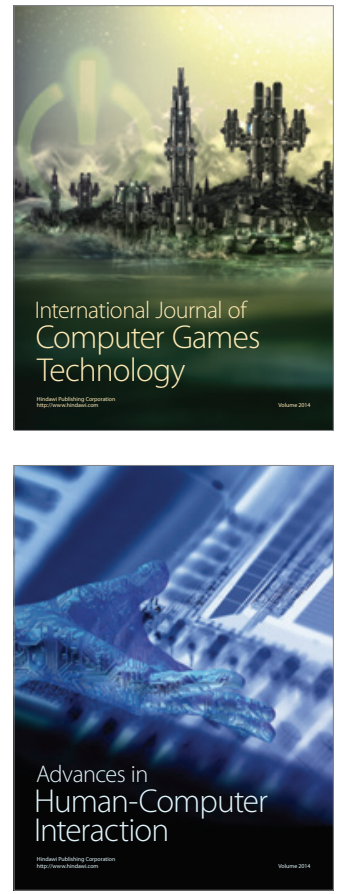
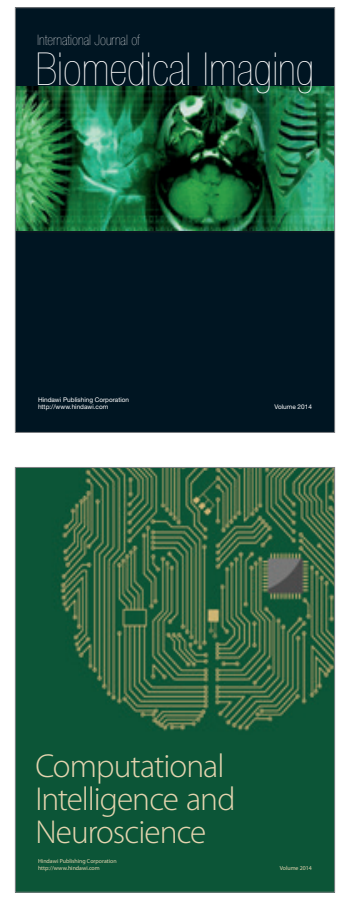
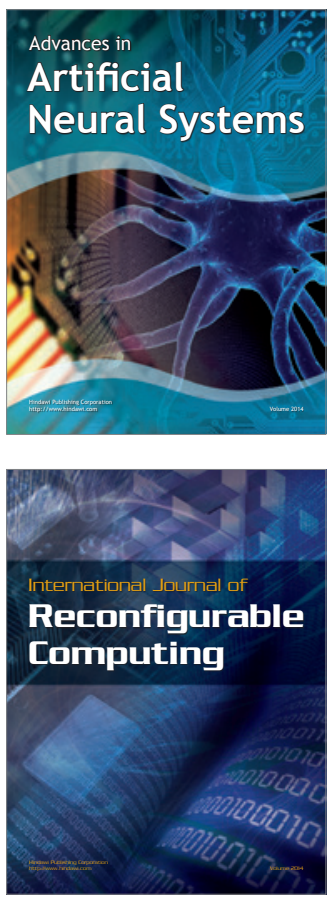
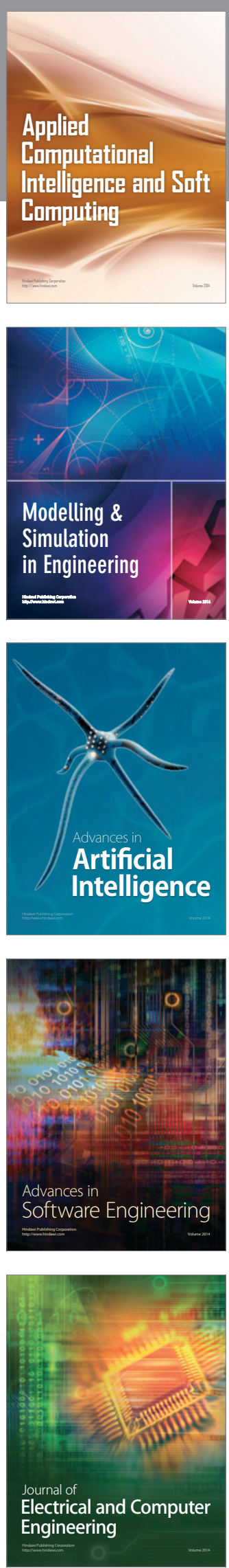